\title{
Computational comparison of quantum-mechanical models for multistep direct reactions
}

\author{
A. J. Koning and J. M. Akkermans \\ Netherlands Energy Research Foundation, 1755 ZG Petten, The Netherlands
}

(Received 5 June 1992)

\begin{abstract}
We have carried out a computational comparison of all existing quantum-mechanical models for multistep direct (MSD) reactions. The various MSD models, including the so-called FeshbachKerman-Koonin, Tamura-Udagawa-Lenske and Nishioka-Yoshida-Weidenmüller models, have been implemented in a single computer system. All model calculations thus use the same set of parameters and the same numerical techniques; only one adjustable parameter is employed. The computational results have been compared with experimental energy spectra and angular distributions for several nuclear reactions, namely, ${ }^{90} \mathrm{Zr}\left(p, p^{\prime}\right)$ at $80 \mathrm{MeV},{ }^{209} \mathrm{Bi}\left(p, p^{\prime}\right)$ at $62 \mathrm{MeV}$, and ${ }^{93} \mathrm{Nb}\left(n, n^{\prime}\right)$ at $25.7 \mathrm{MeV}$. In addition, the results have been compared with the Kalbach systematics and with semiclassical exciton model calculations. All quantum MSD models provide a good fit to the experimental data. In addition, they reproduce the systematics very well and are clearly better than semiclassical model calculations. We furthermore show that the calculated predictions do not differ very strongly between the various quantum MSD models, leading to the conclusion that the simplest MSD model (the Feshbach-Kerman-Koonin model) is adequate for the analysis of experimental data.
\end{abstract}

PACS number(s): 24.60.Gv, 25.40.Ep, 25.40.Fq

\section{INTRODUCTION}

In nucleon-induced nuclear reactions, one conventionally distinguishes between fast, direct processes and slow, compound processes. However, for reactions with incident energies between 10 and $200 \mathrm{MeV}$ per nucleon, it is found that emission can take place after the direct stage but long before the attainment of statistical equilibrium in the compound stage. Such processes are called preequilibrium reactions and account for a significant fraction of the total reaction cross section. Quantummechanical preequilibrium theory distinguishes between multistep compound (MSC) processes (i.e., all particles remain bound throughout the successive stages of the reaction) and multistep direct (MSD) reactions (i.e., at least one particle remains in the continuum). In Ref. [1] we have analyzed the relationships between the various existing multistep direct reaction models, including the models of Feshbach, Kerman, and Koonin (FKK) [2], Tamura, Udagawa, and Lenske (TUL) [3], and Nishioka, Yoshida, and Weidenmüller (NWY) [4]. The step that we take in the present paper is to perform a practical computational comparison between these MSD models, so that conclusions can be drawn about their validity and applicability. This work is the first to give a comprehensive computational intercomparison of the various quantum MSD models. Up to now, only a few, isolated calculations have been carried out [3,6-8]. Accordingly, we have implemented the mentioned MSD models in a single computer program called KAPSIES in order to compare them with each other and with experimental data. This integration into one system has the obvious advantage that the same set of parameters and the same set of numerical techniques can be used for the calculation of the cross sec- tions. Consequently, observed differences can be said to be of a purely theoretical nature and not the result of an inconsistent comparison between two different computer programs. Another attractive feature of such a MSD reaction code is that if the corresponding models can be implemented in an economical way, they can eventually serve as physically better justified quantum-mechanical alternatives to the classical preequilibrium models that dominate the nuclear data applications nowadays $[9,10]$.

The most reliable evidence that the MSD reaction mechanism may be important is the presence of smooth forward peaked angular distributions over a wide range of outgoing energies. Furthermore, since the MSC mechanism is restricted to the low energy end of the preequilibrium region, the total relative contribution of MSD processes to the preequilibrium cross section increases with increasing incident energy. At high incident energies (above, say, $50 \mathrm{MeV}$ ) the MSD process accounts for virtually all of the preequilibrium cross section and the MSC mechanism only participates as a part of the MSDMSC crossover effect. In analogy with the theoretical derivations of the various MSD models, we have pursued a consistent extrapolation to the continuum of the direct reaction methods that are generally used to predict cross sections for discrete states.

In Sec. II, the theoretical results of Ref. [1] are recapitulated and written in a form suitable for computer applications. In Sec. III, we discuss the method of performing distorted wave Born approximation (DWBA) calculations in the continuum. Next, in Sec. IV a brief description of the calculation of MSD cross sections is given. In Sec. V, the various MSD models are compared with the experimental data and with the Kalbach systematics [11]. Finally, some conclusions of this paper are given. 


\section{MULTISTEP DIRECT CROSS SECTIONS}

Since the mathematical derivation of the different MSD models has been extensively discussed in Ref. [1], we will only list the various cross section formulas. However, in contrast with Ref. [1], we retain all kinematical factors in the expressions for the cross section, so that these are immediately suitable for computational implementation.

As seen in Ref. [1], the various MSD models use different quantum-statistical assumptions, called leadingparticle statistics and residual-system statistics. The TUL and NWY models only employ the latter, whereas the FKK model basically uses the first. Both types of statistics enable the continuum cross section to be expressed as an incoherent sum of one-step and multistep cross sections. The double-differential energy-averaged one-step cross section turns out to be the same for all MSD models. It can be written as the product of the state density and a squared matrix element averaged over particle-hole states. Decomposition into different transferred angular momenta $l$ then yields a weighted sum of first-order DWBA cross sections (averaged for each $l$ value):

$$
\frac{d^{2} \sigma^{(1)}\left(E, \Omega \leftarrow E_{0}, \Omega_{0}\right)}{d \Omega d E}=\sum_{l}(2 l+1) R(l) \omega_{1 \mathrm{p} 1 \mathrm{~h}}\left(E_{0}-E\right)\left[\frac{d \sigma^{(1)}\left(E, \Omega \leftarrow E_{0}, \Omega_{0}\right)}{d \Omega}\right]_{l}^{\mathrm{DWBA}}
$$

where $E_{0}, \Omega_{0}$ and $E, \Omega$ are the incident and outgoing energy and angle, respectively. The function $R(l)$ (with spin cutoff factor $\sigma$ ) represents the spin distribution of the residual states in the continuum. It is given by

$$
R(l)=\frac{2 l+1}{2 \sqrt{2 \pi} \sigma^{3}} \exp \left[-\frac{\left(l+\frac{1}{2}\right)^{2}}{2 \sigma^{2}}\right]
$$

and satisfies the relation $\sum_{l}(2 l+1) R(l)=1$. The Williams particle-hole state density for $p$ particles and $h$ holes is [12]

$$
\omega_{p, h}\left(E_{x}\right)=g^{n} \frac{\left(E_{x}-A_{p, h}-P\right)^{n-1}}{p ! h !(n-1) !},
$$

where $g$ is the single-particle state density, $E_{x}$ the excitation energy, $n=p+h$ the exciton number, $A_{p, h}=$ $[p(p-1)+h(h-1)] / 4$ the Pauli correction factor, and $P$ the pairing correction.
In general, the one-step cross section provides the major part of the total (outgoing energy-integrated) cross section. Especially at the highest outgoing energies the first step is predominant and by itself practically sufficient to explain the data. Nevertheless, interesting aspects of the MSD model comparison are found in the higher step processes. As outlined in Ref. [1], different types of statistical postulates generate a variety of two-step direct cross sections. Among these, the FKK model is the most appealing from the computational point of view. The original FKK expression as given by Eqs. (4.21) and (4.22) of Ref. [2] involves doubledifferential transition probabilities and state densities of the continuum particle. Upon choosing a representation in terms of the intermediate energy $E_{1}$ and intermediate solid angle $\Omega_{1}$, we can rewrite this expression in a compact recursive form which enables a very fast computation of the second- and higher-step cross sections. In particular, the FKK two-step cross section reads

$$
\frac{d^{2} \sigma^{(2)}\left(E, \Omega \leftarrow E_{0}, \Omega_{0}\right)}{d \Omega d E}=\frac{m}{4 \pi^{2} \hbar^{2}} \int d \Omega_{1} \int d E_{1} E_{1} \frac{d^{2} \sigma^{(1)}\left(E, \Omega \leftarrow E_{1}, \Omega_{1}\right)}{d \Omega d E} \frac{d^{2} \sigma^{(1)}\left(E_{1}, \Omega_{1} \leftarrow E_{0}, \Omega_{0}\right)}{d \Omega_{1} d E_{1}},
$$

where $m$ is the reduced nucleon mass and the continuum one-step cross sections are given by Eq. (1). This can easily be generalized to the $n$-step cross section:

$$
\begin{aligned}
\frac{d^{2} \sigma^{(n)}\left(E, \Omega \leftarrow E_{0}, \Omega_{0}\right)}{d \Omega d E}=\frac{m}{4 \pi^{2} \hbar^{2}} \int d \Omega_{n-1} \int & d E_{n-1} E_{n-1} \frac{d^{2} \sigma^{(1)}\left(E, \Omega \leftarrow E_{n-1}, \Omega_{n-1}\right)}{d \Omega d E} \\
& \times \frac{d^{2} \sigma^{(n-1)}\left(E_{n-1}, \Omega_{n-1} \leftarrow E_{0}, \Omega_{0}\right)}{d \Omega_{n-1} d E_{n-1}} .
\end{aligned}
$$

A conspicuous feature of the convolution structure of the FKK model is that the $n$-step cross section can be expressed in terms of the result of the previous stage and is therefore easy to calculate. Basically, only first-order DWBA cross sections are required as input for the calculation of the multistep cross section.

Whereas the application of leading-particle statistics achieves the separation of a two-step cross section into two independent one-step cross sections, residual-system statistics yields two-step expressions that remain dependent on full second-order DWBA. As explained in Ref. [1], this is a natural result of the failure of residual-system statistics to remove the interference effects associated with the leading particle. The two examples of residualsystem statistics are the TUL model [3] and the NWY model [4]. The TUL model applies residual-system statis- 
tics after both the first and second steps (adiabatic approximation). This is equivalent to saying that at each stage of the reaction there occurs random configuration mixing of the particle-hole states over the real nuclear states before the creation of a new particle-hole pair. The true state densities that accompany residual-system statistics provide an effective description of this process.
In the limit of vanishing residual interaction, the true state density becomes equal to the particle-hole model level density. With this approximation, the TUL energyaveraged two-step cross section takes a global form and can be written as a weighted sum over second-order DWBA cross sections:

$$
\begin{aligned}
\frac{d^{2} \sigma^{(2)}\left(E, \Omega \leftarrow E_{0}, \Omega_{0}\right)}{d \Omega d E}=\sum_{l_{1}, l_{2}} \int & d E_{1}\left(2 l_{2}+1\right) R\left(l_{2}\right) \omega_{1 \mathrm{p} 1 \mathrm{~h}}\left(E_{1}-E\right)\left(2 l_{1}+1\right) R\left(l_{1}\right) \\
& \times \omega_{1 \mathrm{p} 1 \mathrm{~h}}\left(E_{0}-E_{1}\right)\left[\frac{d \sigma^{(2)}\left(E, \Omega \leftarrow E_{1}, \Omega_{1} \leftarrow E_{0}, \Omega_{0}\right)}{d \Omega}\right]_{l_{1}, l_{2}}^{\mathrm{DWBA}},
\end{aligned}
$$

where $l_{1}$ and $l_{2}$ are the angular momenta transferred in the first and second steps, respectively. Upon comparison of Eq. (6) with the FKK expression (4) we observe that leading-particle statistics is a more drastic approximation than residual-system statistics. In the latter case, the dependency on the full second-order DWBA cross section remains.

In the NWY model, it is assumed that the intermediate process occurs so fast that configuration mixing at the first stage does not occur and, instead, the system immediately proceeds with the creation of an additional particle-hole pair. Consequently, residual-system statistics only applies to the final states (sudden approximation). As a result, the corresponding two-step cross section formula is quite complicated since it involves the coherent summation of four alternative paths leading to the final $2 \mathrm{p} 2 \mathrm{~h}$ state. Hence, we do not obtain a simple expression in terms of individual second-order DWBA cross sections. An exact calculation of the NWY two-step cross section would require microscopic information about the model states and an operation in an existing direct reaction code that properly isolates the two-step amplitudes and subsequently sums them as prescribed in Ref. [4], a task which is very difficult to perform. Nevertheless, we can obtain an estimate of the NWY model by assuming that the individual amplitudes do not differ significantly and thus add constructively to the cross section. This is however an upper limit and therefore may overestimate a proper microscopic calculation of the NWY cross sections. Then, our approximation of the NWY two-step cross section becomes

$$
\frac{d^{2} \sigma^{(2)}\left(E, \Omega \leftarrow E_{0}, \Omega_{0}\right)}{d \Omega d E}=16 \sum_{l_{1}, l_{2}}\left(2 l_{2}+1\right) R\left(l_{2}\right) \omega_{2 \mathrm{p} 2 \mathrm{~h}}\left(E_{0}-E\right)\left(2 l_{1}+1\right) R\left(l_{1}\right) \overline{\left[\frac{d \sigma^{(2)}\left(E, \Omega \leftarrow E_{1}, \Omega_{1} \leftarrow E_{0}, \Omega_{0}\right)}{d \Omega}\right]_{l_{1}, l_{2}}^{\mathrm{DWBA}}}
$$

where the bar on the right-hand side denotes an average over the intermediate energy. Having performed the aforementioned approximation, the most prominent difference with the TUL model is (apart from the factor of 16 resulting from our additional assumption) that in the NWY model there is no statistical weighting (state density) after the first step, but instead a $2 \mathrm{p} 2 \mathrm{~h}$ state density at the final stage only.

The computational comparison that follows is based on a direct implementation of the formulas given in this section. An important additional approximation on the original models that we like to point out here is the application of the Williams state density formula in all models. Although the MSD models associated with residualsystem statistics are based on random configuration mixing and therefore necessarily involve true level densities [5], we have simplified this by taking the limit of vanishing residual interaction. The advantage of using the
Williams state density formula in all MSD models is that it enables a consistent comparison of nuclear dynamics involving residual-system statistics against nuclear dynamics involving leading-particle statistics.

\section{DWBA IN THE CONTINUUM}

The formulas given in the previous section clearly reveal that a sizable part of the calculation consists of computing double-differential DWBA cross sections in the continuum for several energies and transferred angular momenta. When these are calculated they can be processed (i.e., multiplication by level densities and integration over intermediate energy) according to the several aforementioned MSD prescriptions. In this way the continuum cross sections are obtained.

The general method is exemplified by expression (1) for the one-step cross section in combination with Fig. 1. 

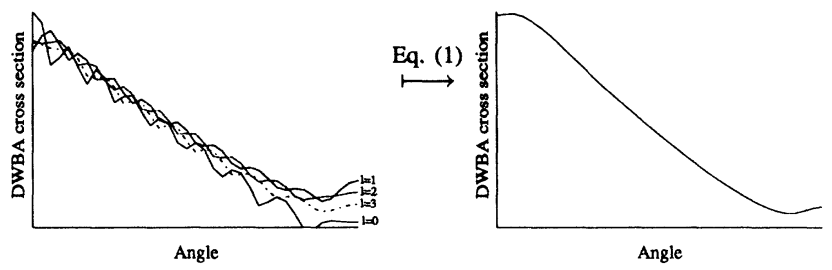

FIG. 1. Smooth forward peaked angular distribution as a result of the statistical averaging. DWBA cross sections for the fictitious states $0^{+}, 1^{-}, 2^{+}, 3^{-}$are calculated for the reaction $\left(p, p^{\prime}\right)$ on ${ }^{90} \mathrm{Zr}$ at $80 \mathrm{MeV}$ of incident energy and 60 $\mathrm{MeV}$ of outgoing energy and subsequently averaged according to Eq. (1).

DWBA cross sections are computed at a specified excitation energy for each residual spin. After multiplication with the corresponding angular momentum dependent state density and summation over all angular momenta the (average) MSD cross section per $\mathrm{MeV}$ is obtained. The result is that the typical forward-backward asymmetry remains, whereas the individual oscillatory behavior of the angular distribution of each residual state disappears, as depicted in Fig. 1. Hence, we find a smooth forward peaked angular distribution. This implies that the individual DWBA cross sections, not being related to a specific state, merely serve as a global representative of the dynamical process. The statistical properties of the nucleus (i.e., the state density) assure that their individual characteristics average out.

The first microscopic MSD application has been performed by Bonetti et al. $[6,7]$ for an implementation of the FKK model. In this approach, a simple spherical Nilsson model (which is of sufficient sophistication because of the lack of knowledge of the detailed level properties in the continuum) has been used to generate the $1 \mathrm{p} 1 \mathrm{~h}$ states. These are grouped into several classes, each class corresponding to the same total angular momentum and region of excitation energy. For a randomly chosen sample from the particle-hole states, the DWBA angular distributions are calculated using microscopic twoparticle form factors (the hole is treated as a particle). The effective nucleon-nucleon interaction is represented by a Yukawa potential with strength $V_{0}$ and range $1 \mathrm{fm}$ and the bound-state wave functions are generated with a real Woods-Saxon potential. Subsequently, the resulting angular distributions are averaged over the number of states in each class so that an average DWBA cross section per $l$ value and excitation energy is obtained and the FKK cross sections can be computed. Finally, the strength $V_{0}$ can be adjusted to reproduce the data and be compared with independent estimates [13]. A disadvantage of this method is that in the neighborhood of a certain excitation energy few or no particle-hole states may be available. Generally, the samples taken are rather small. Then, in order to obtain a reliable average, distant particle-hole states have to be included in the sample. Consequently, the correspondence between the true excitation energy and the energies of the shell model states is lost. Furthermore, as observed by Marcinkowski et al. [8], if some of the DWBA angular distributions deviate very much from the behavior of the others in the same class, the validity of the average upon taking too small a sample becomes dubious. Hence, this method requires many microscopic DWBA calculations of the same type to obtain only one averaged DWBA cross section. For this reason, it is impracticable to perform a proper microscopic calculation of the TUL and NWY two-step cross sections, since that would require about $10000 \mathrm{mi}-$ croscopic second-order DWBA calculations.

An alternative, macroscopic approach has been employed by Tamura et al. [3]. These authors found that an average (over many shell model states) of microscopic form factors of the same total angular momentum yields an average form factor that is surface peaked. Thus, they concluded that each average DWBA cross section appearing in the MSD formulas can equally well be determined by one macroscopic calculation. In this case, the macroscopic form factor is related to the derivative of the optical potential $U(r)$ :

$$
F_{l}(r)=\beta_{l} R_{0} \frac{d U(r)}{d r}
$$

where $R_{0}$ is the nuclear radius. The dynamical deformation parameter for multipole excitations $\beta_{l}$, which is a direct measure for the interaction strength, serves as the adjustable factor. For direct reactions to the continuum, the value of $\beta_{l}$ is, of course, much smaller than the values commonly encountered in reactions to strongly coupled states. As an additional verification, we have checked that comparison of averaged microscopic DWBA cross sections (sampled over randomly chosen particle-hole states with the same total angular momentum) and the corresponding macroscopic DWBA cross sections does not exhibit significant differences. An example of this verification can be found in Sec. V A. Therefore, we have also used the macroscopic DWBA approach of Ref. [3] for our MSD calculations. With this method, consistency is maintained with the analysis of discrete direct reactions, where the macroscopic form factor (8) is frequently applied to explain the bulk of the experimentally observed levels. The practical advantage is that the number of calculations is significantly smaller than in the microscopic case, without a reduction in quality.

\section{COMPUTATIONAL COMPARISON}

Having established the theoretical differences between the various MSD models and the method to calculate the necessary DWBA cross sections, we can proceed with the implementation of the MSD expressions and compare them with each other and with the experimental data. To this end, we have constructed the code system KAPSIES (see Fig. 2) which calculates MSD cross sections as given by Eqs. (1)-(7).

Later on, we will also compare our results with two other implementations of MSD models, namely, the code by Bonetti and Chiesa [14] for the FKK model and the code by Tamura et al. [15] for the TUL model. The NWY model has not been implemented before. The present work is the first attempt to perform a MSD model com- 


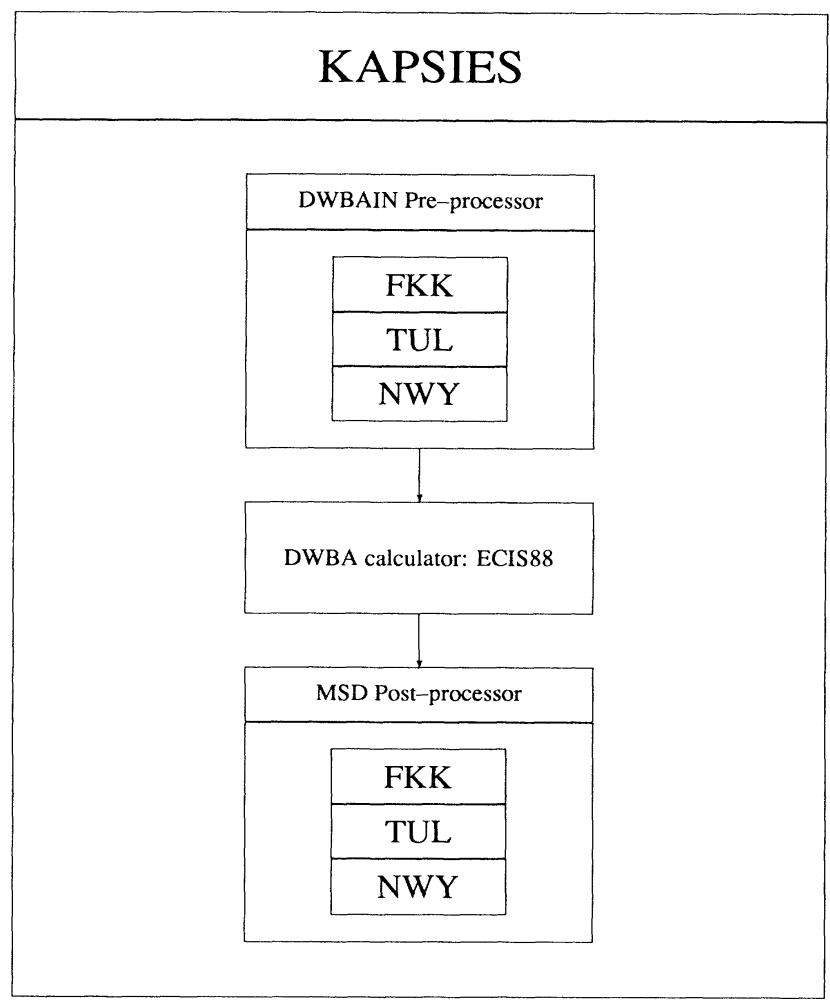

FIG. 2. General overview of code system KAPSIES.

parison on a consistent basis, i.e., all different models are implemented in one computer code, with a single set of physical parameters.

As mentioned before, by far the largest part of the computation is taken by the DWBA calculations. In KAPSIES, this task is performed by the coupled channels code ECIS88 of Raynal [16]. This choice was motivated by the general success of ECIS 88 in predicting direct reaction cross sections for discrete states and its capability of handling a large variety of reaction types over a wide energy range. Of course, a full coupled channels calculation is too detailed and unnecessary for our purposes. Fortunately, the coupled channels equations in ECIS88 are calculated by sequential iteration, as described in detail in Refs. [16,17]. Accordingly, setting the number of iterations equal to one enables a relatively fast computation of first- and higher-order DWBA cross sections which provide the input for the MSD expressions. Besides this DWBA calculator, our code system consists of two further programs. Firstly, we have constructed a preprocessing program DWBAIN that generates the input files for ECIS88. For each intermediate and final energy and transferred angular momentum, the DWBA cross section must be calculated. As far as the TUL and NWY models are concerned, we have restricted ourselves to secondorder DWBA only because inclusion of higher orders is too time consuming.

The initial, intermediate, and final distorted waves are calculated using global optical potential systematics. The same optical potential is also used for the cal- culation of the form factor, which is done according to Eq. (8). The $\beta_{l}$ are taken independent of $l$; therefore the index $l$ will be dropped in the remainder of this paper. With this information, for each point on the energy grid an input file is created for the calculation of the DWBA cross section for each transferred angular momentum.

After the DWBA calculation by ECIS88 the DWBA cross sections are processed by the post-processing program MSD to yield the continuum MSD (doubledifferential and integrated) cross sections. These are obtained by multiplying the DWBA cross sections by the state density, summation over angular momenta and energy integration according to Eqs. (1)-(7). For the state density parameters we used standard parametrizations. The single-particle state density $g$ is

$$
g=\frac{A}{13}
$$

The default pairing correction is [18]

$$
\begin{aligned}
P & =25.6 A^{-\frac{1}{2}}, \quad N \text { even, } Z \text { even, } \\
& =12.8 A^{-\frac{1}{2}}, \quad A \text { odd, } \\
& =29.4 A^{-1}, \quad N \text { odd, } Z \text { odd, }
\end{aligned}
$$

and for the spin cutoff factor we use [19]

$$
\sigma^{2}=0.24 n A^{\frac{2}{3}},
$$

where $n$ is the exciton number.

In order to compare the calculations with the experimental data, we assume that at the highest outgoing energy of the grid the cross section is entirely dominated by the multistep direct mechanism. Accordingly, we have adjusted $\beta$ so that at this outgoing energy the magnitude of the MSD cross section reproduces the experimental data. This single adjustment then completely determines the results for the lower outgoing energies.

As an additional test for the validity of the quantummechanical models, we compare our results with the systematics of Kalbach, a purely phenomenological approach based on fitting a large number of experimental continuum angular distributions [11]. This parametrization generally provides an adequate fit to the shape of the preequilibrium angular distributions and is therefore frequently employed in nuclear data applications (see, for example, Ref. [20]). Kalbach obtained the following formula for the continuum double-differential cross section:

$$
\begin{aligned}
\frac{d^{2} \sigma}{d \Omega d \epsilon_{b}}=\frac{1}{4 \pi} \frac{d \sigma}{d \epsilon_{b}} \frac{a}{\sinh (a)}[ & \cosh (a \cos \Theta) \\
& \left.+f_{\mathrm{MSD}} \sinh (a \cos \Theta)\right]
\end{aligned}
$$

with

$$
\begin{aligned}
a\left(e_{b}^{\prime}, e_{a}^{\prime}\right)= & 0.04 \frac{E_{1} e_{b}^{\prime}}{e_{a}^{\prime}}+1.8 \times 10^{-6}\left(\frac{E_{1} e_{b}^{\prime}}{e_{a}^{\prime}}\right)^{3} \\
& +6.7 \times 10^{-7} M_{a} m_{b}\left(\frac{E_{3} e_{b}^{\prime}}{e_{a}^{\prime}}\right)^{4}, \\
E_{1}= & \min \left(e_{a}^{\prime}, 130 \pm 10 \mathrm{MeV}\right), \\
E_{3}= & \min \left(e_{a}^{\prime}, 41 \pm 5 \mathrm{MeV}\right), \\
e_{b}^{\prime}= & \epsilon_{b}+S_{b}, \\
e_{a}^{\prime}= & \epsilon_{a}+S_{a} .
\end{aligned}
$$


Here, $\epsilon_{a}$ and $\epsilon_{b}$ are the incident and the outgoing energies, respectively. The number $M_{a}$ (representing the incident particle) is 1 for neutrons, protons, and deuterons and 0 for alpha particles, while $m_{b}$ (representing the outgoing particle) is 1 for protons, deuterons, tritons, and ${ }^{3} \mathrm{He}$ particles, $\frac{1}{2}$ for neutrons and 2 for alpha particles. The Myers and Swiatecki mass formula for the separation energy $S$ can be found in Ref. [11].

The continuum angle-integrated cross section $\frac{d \sigma}{d \epsilon_{b}}$ of Eq. (12) can be written as the sum of a MSC part and a MSD part:

$$
\frac{d \sigma}{d \epsilon_{b}}=\frac{d \sigma_{\mathrm{MSD}}}{d \epsilon_{b}}+\frac{d \sigma_{\mathrm{MSC}}}{d \epsilon_{b}} .
$$

The fraction $f_{\mathrm{MSD}}$ of Eq. (12) represents the contribution of the MSD process. If $f_{\text {MSD }}=0$, Eq. (12) yields a symmetric angular distribution, which is in agreement with the usual assumption about MSC reactions. In this work, we consider MSD cross sections $\left(f_{\mathrm{MSD}}=1\right)$ only. Hence, for our purposes Eq. (12) reduces to

$$
\frac{d^{2} \sigma_{\mathrm{MSD}}}{d \Omega d \epsilon_{b}}=\frac{1}{4 \pi} \frac{d \sigma_{M S D}}{d \epsilon_{b}} \frac{a}{\sinh (a)} \exp (a \cos \Theta) \text {. }
$$

For consistency reasons, we have used the angleintegrated cross sections as obtained from the MSD models as input for Eq. (15).

\section{RESULTS}

Our choice of reactions to be analyzed has been motivated by two considerations. Firstly, we want to investigate the validity of our MSD calculations over a wide mass and energy range. Of course, the experimental evidence that MSD processes provide a significant fraction of the cross section is an essential ingredient for such a study. An indication for this is that the measured reactions are characterized by forward peaked, smooth angular distributions and by high energy tails in the outgoing energy spectrum. This requirement is fulfilled for incident energies between 10 and $200 \mathrm{MeV}$. Secondly, we want to compare our results with other published analyses and discuss the differences, both from a physical and a computational point of view. With this in mind, we have chosen the $80 \mathrm{MeV}\left(p, p^{\prime}\right)$ reaction on ${ }^{90} \mathrm{Zr}$, the $62 \mathrm{MeV}\left(p, p^{\prime}\right)$ reaction on ${ }^{209} \mathrm{Bi}$, and the 25.7 $\mathrm{MeV}\left(n, n^{\prime}\right)$ reaction on ${ }^{93} \mathrm{Nb}$ for our comparison. These reactions have been analyzed before by both quantummechanical [21] and classical [22,23] approaches and in totality they cover an interesting region of mass and energy. In the following, the calculated double-differential and angle-integrated cross sections for these reactions are tested against experiment and against the systematics of Kalbach.

An additional remark concerns the TUL and NWY models. As explained before, it is practically impossible to calculate the continuum three-step direct cross sections for these models. Our calculations suggest that this deficiency can be neglected for the total (outgoingenergy integrated) cross section and the cross sections at high outgoing energies, where the first step is pre- dominant. The contribution of the second step becomes sizable for cross sections at low outgoing energies (at all angles) or at backward angles (at all outgoing energies). In general, the three-step contribution still does not contribute significantly to the cross section for these cases. However, for the special combination of low outgoing energies and backward angles, the three-step cross section may no longer be negligible (although it is seldom dominant). Only in this case the experimental angular distributions may be underestimated if only the first and second step are included in the calculation. To have an estimate for this, we have supplemented the NWY and TUL cross sections with the third and fourth steps of the FKK model. It will soon become clear from our results that this addition only has some effect in the mentioned special case. The general insignificance of the third and higher steps leaves all other typical aspects of the various models unaffected.

For the aforementioned three reactions, we will present an analysis of the angular distribution at three different outgoing energies. Furthermore, the three different twostep cross sections are compared at these energies and we give the relative contribution of each step of the MSD reaction. If a sufficient number of experimental angleintegrated cross sections is available, these will also be compared with our calculations.

\section{A. $80 \mathrm{MeV}\left(p, p^{\prime}\right)$ on ${ }^{80} \mathrm{Zr}$}

The $80 \mathrm{MeV}$ inelastic proton scattering experiment on ${ }^{90} \mathrm{Zr}$ has been performed by Cowley et al. and analyzed by the FKK model computer code MUDIR of Bonetti. Both the experimental and computational results were reported in Ref. [21]. Here, we perform a reanalysis of this reaction with our own code system. We present the energy-averaged MSD cross sections according to the FKK, TUL, and NWY models at outgoing energies of 60 , 40 , and $20 \mathrm{MeV}$.

We used the global optical potential of Becchetti and Greenlees for the incident, intermediate, and the outgoing channels as well as for the calculation of the form factor. For comparison, the state density parameters were taken the same as that of Ref. [21], i.e., $\sigma=2.3, P=0$, and $a=10.5 \mathrm{MeV}^{-1}\left(g=\frac{6 a}{\pi^{2}}=6.38 \mathrm{MeV}^{-1}\right)$. Figures 3-5 show that all models provide a good fit to the MSD angular distributions. In particular, the FKK and NWY curves at high outgoing energies are in excellent agreement with the data. Apparently, the systematics of Kalbach can even be slightly improved by the quantummechanical preequilibrium models. For all models, the same value for the deformation parameter, $\beta=0.00490$, was used. At the lowest outgoing energy, the calculated MSD cross sections underestimate the experimental data, although the shape of the angular distribution is still in reasonable agreement with the measurement. This underprediction is to be expected, since the role of other competing processes will become non-negligible at low outgoing energies. Firstly, when the difference between incident and outgoing energies is large, multiple emission may occur because further protons can be emitted from the highly excited residual nucleus. Secondly, the contri- 
bution of the MSD-MSC crossover effect may be considerable. Several independent estimates indicate that the multistep compound mechanism is important for the incident energy region between 10 and $20 \mathrm{MeV}$. Hence, the pure MSC process (in which the propagation of the system through the bound configurations of the compound nucleons already starts at the first stage) does not compete with MSD for this particular reaction. However, we would expect that the leading particle loses a large fraction of its initial kinetic energy after one or more successive collisions in a multistep process. Then, instead
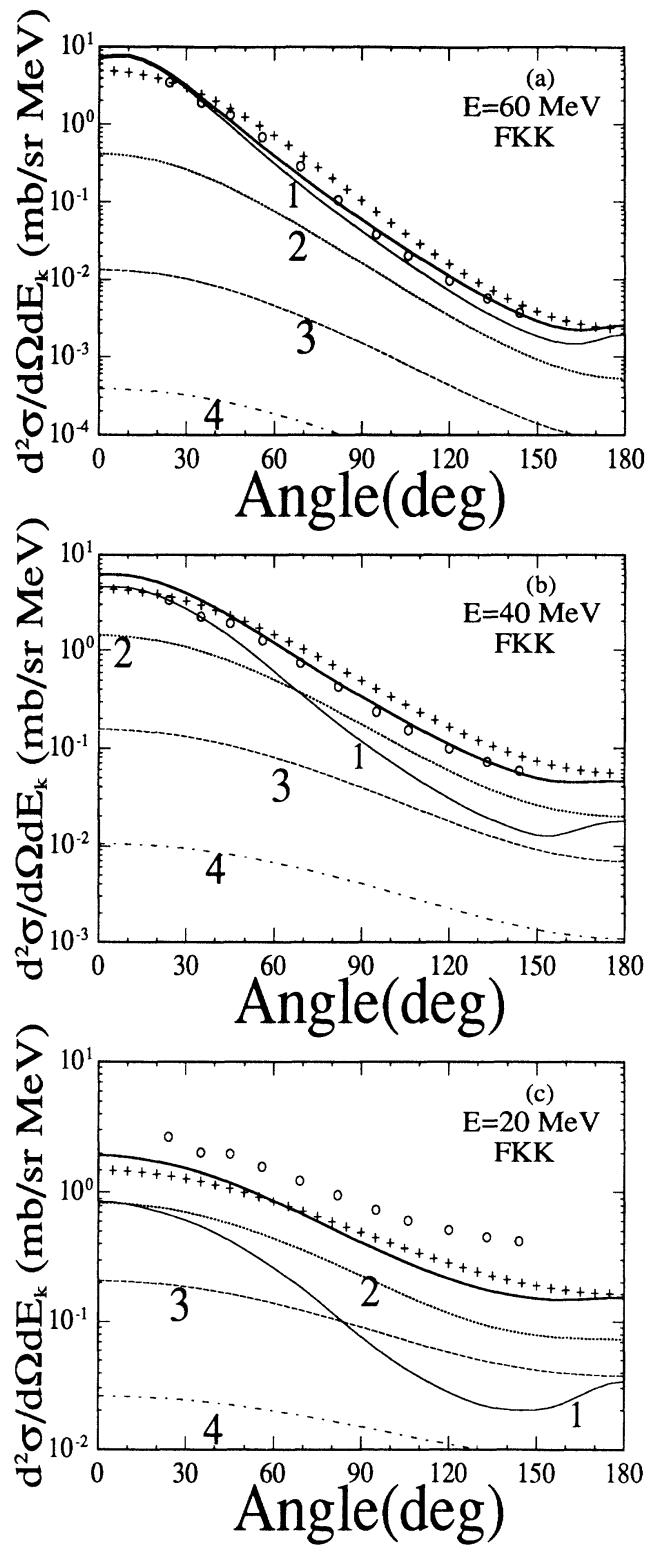

FIG. 3. MSD cross sections according to the FKK model for $80 \mathrm{MeV}\left(p, p^{\prime}\right)$ on ${ }^{90} \mathrm{Zr}$ at outgoing energies of (a) $60 \mathrm{MeV}$, (b) $40 \mathrm{MeV}$, and (c) $20 \mathrm{MeV}$. The thick solid line represents the total MSD cross section (summed over steps), the circles the experimental data, and the pluses the systematics of Kalbach. of the process of fast emission, some of the particle flux may flow into the bound chain and give rise to multistep compound or compound emission. For the outgoing energy of $20 \mathrm{MeV}$ this MSD-MSC crossover effect certainly has to be taken into account. Very recently, a theory has been constructed for the MSD-MSC crossover mechanism [24] but practical calculations have not (yet) been carried out.

The importance of the individual higher-step contributions may differ from model to model. In Fig. 6, we have isolated the various two-step cross sections and compared
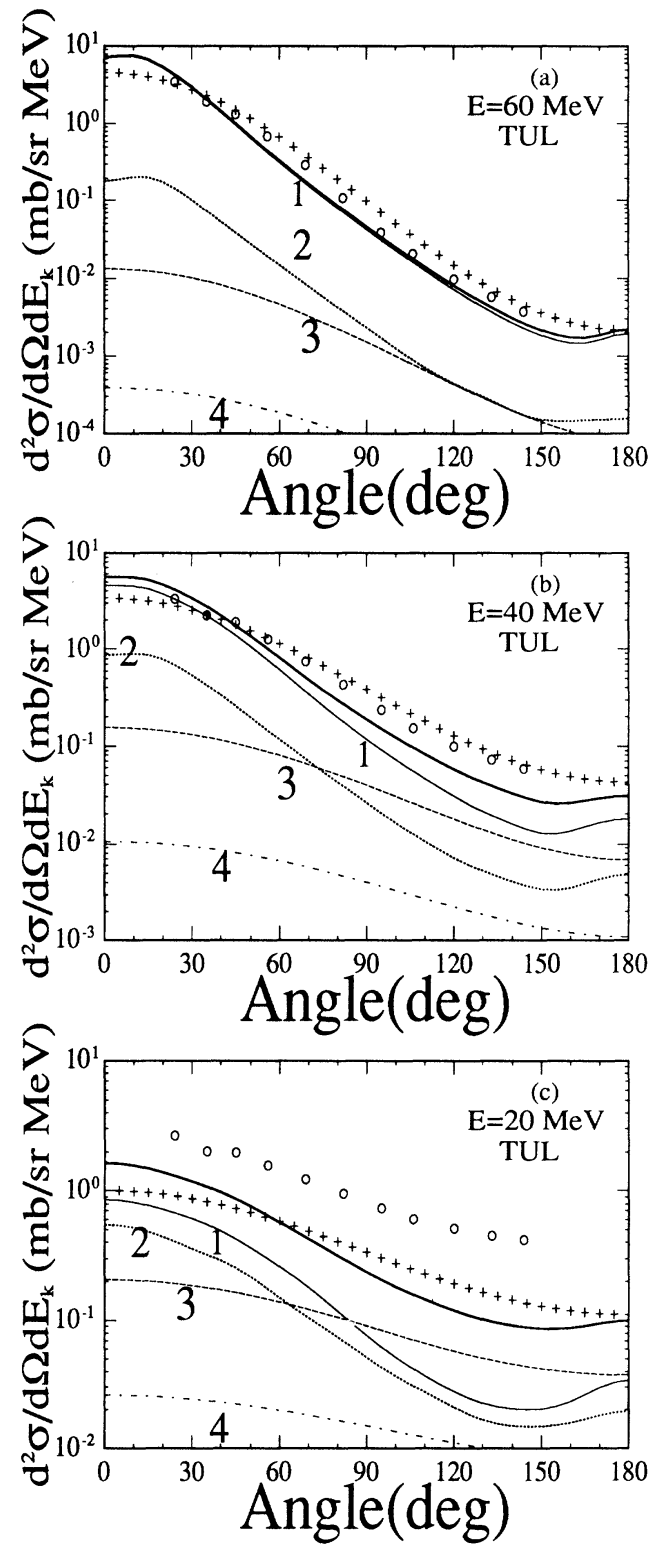

FIG. 4. MSD cross sections according to the TUL model for $80 \mathrm{MeV}\left(p, p^{\prime}\right)$ on ${ }^{90} \mathrm{Zr}$ at outgoing energies of (a) $60 \mathrm{MeV}$, (b) $40 \mathrm{MeV}$, and (c) $20 \mathrm{MeV}$. The thick solid line represents the total MSD cross section (summed over steps), the circles the experimental data, and the pluses the systematics of Kalbach. 
them at the three outgoing energies. These figures reveal the relationship between convolution-type cross sections (leading-particle statistics) and cross sections based on the full second-order DWBA (residual-system statistics). The latter exhibit a more pronounced structure at the very forward and backward angles. This coincides with our expectations from the theory: individual characteristics will be removed more drastically by leadingparticle statistics (which concerns the residual nucleus + fast particle) than residual-system statistics (which only applies to the residual nucleus). Accordingly, a convo-
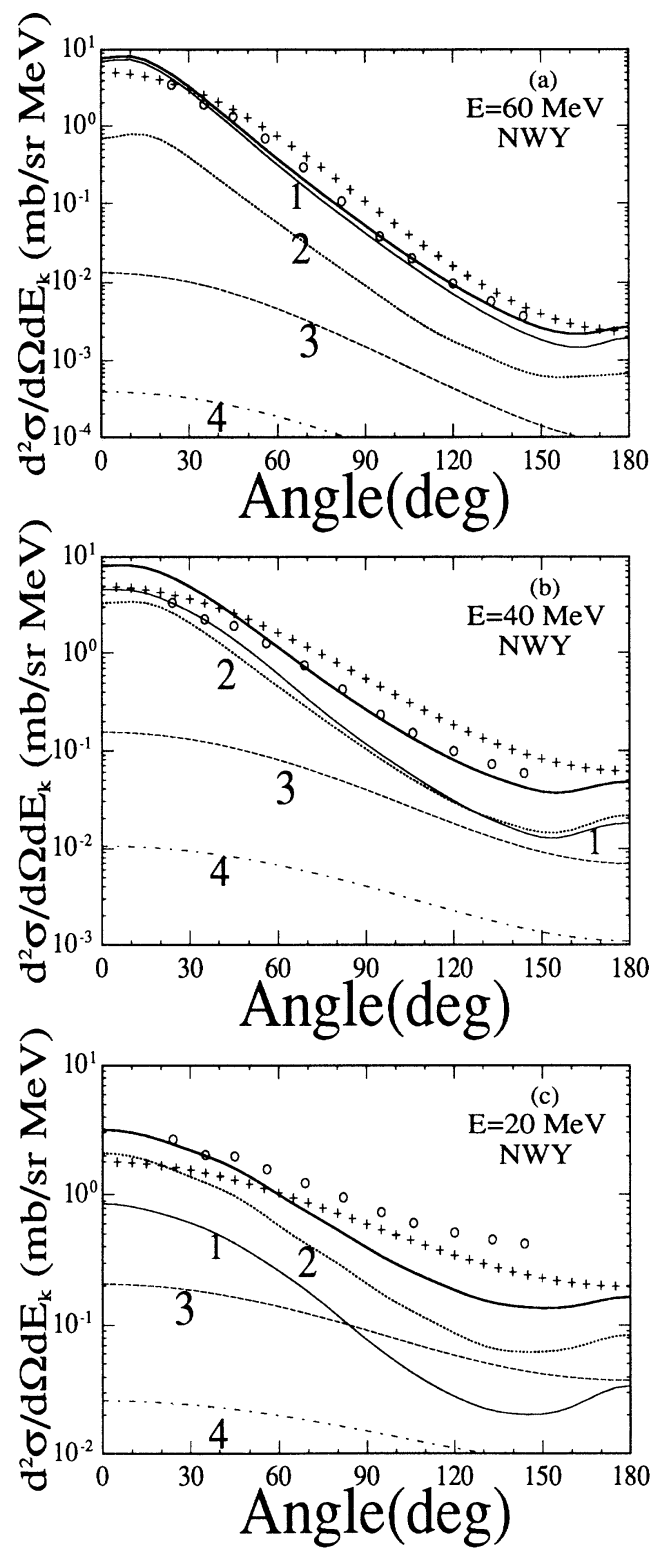

FIG. 5. MSD cross sections according to the NWY model for $80 \mathrm{MeV}\left(p, p^{\prime}\right)$ on ${ }^{90} \mathrm{Zr}$ at outgoing energies of (a) $60 \mathrm{MeV}$, (b) $40 \mathrm{MeV}$, and (c) $20 \mathrm{MeV}$. The thick solid line represents the total MSD cross section (summed over steps), the circles the experimental data, and the pluses the systematics of Kalbach. lution cross section of the FKK type will be more flat than the other two which comprise a summation over individual second-order DWBA cross sections. An additional observation that can be made is that the relative differences between the two-step cross sections remain approximately the same over the whole outgoing-energy range. In particular, the NWY and FKK models are very similar in magnitude, irrespective of the outgoing energy. Of course, all these (dis)similarities are insignificant at high outgoing energies where the model independent first step alone is almost completely responsible for the total cross section. Nevertheless, the importance of the differences between the various multistep contributions increases with decreasing outgoing energies.
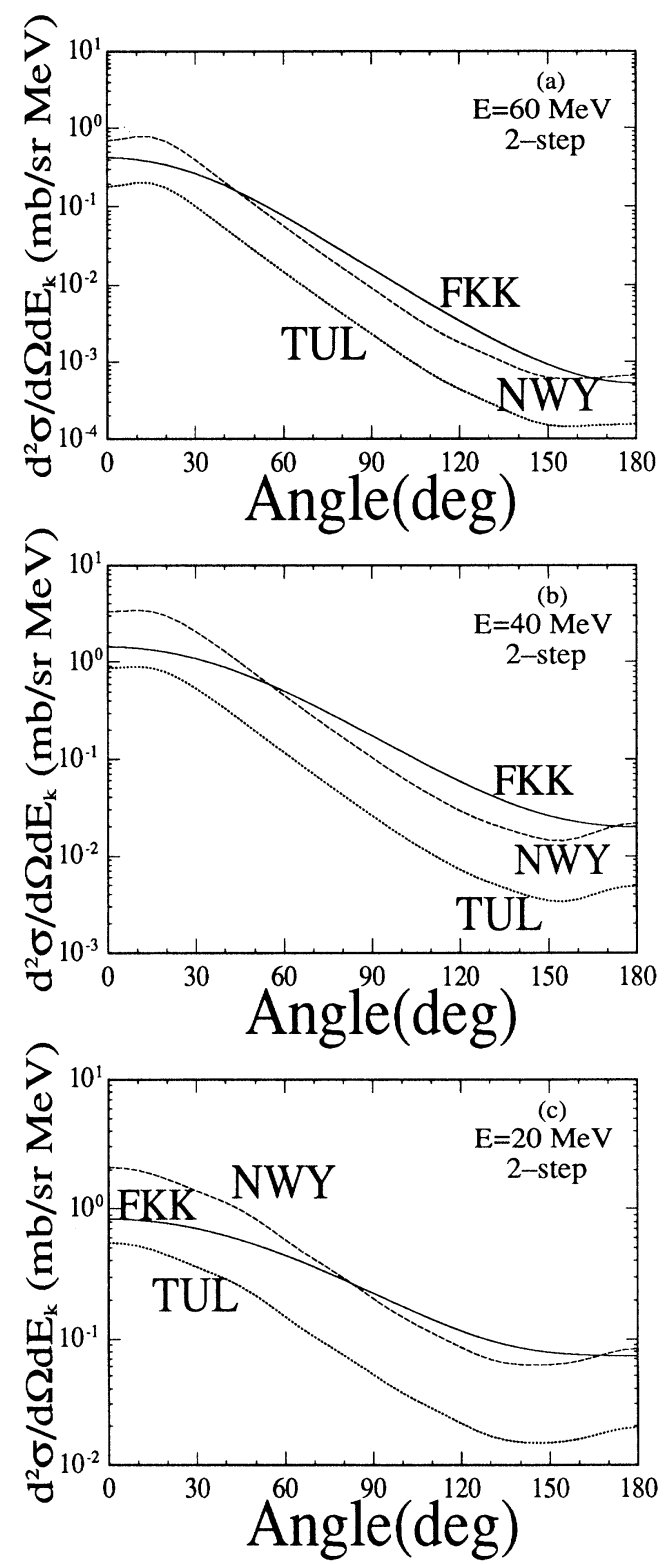

FIG. 6. Two-step cross sections for $80 \mathrm{MeV}\left(p, p^{\prime}\right)$ on ${ }^{90} \mathrm{Zr}$ at outgoing energies of (a) $60 \mathrm{MeV}$, (b) $40 \mathrm{MeV}$, and (c) 20 $\mathrm{MeV}$. 
In Figs. 7 and 8, the angle-integrated and energyintegrated relative contributions of each step are given for each model. They display an alternative confirmation of our statements about the relative importance of the various steps. For this reaction, the MSD part of the spectrum is almost completely dominated by the first step. Higher-step contributions may be crucial in the backward hemisphere, but at these angles the contribution to the angle-integrated cross section is negligible. Integrating the angle-integrated relative contributions over outgoing energy yields the partition of the total MSD cross section into steps. These fractions are displayed in Table I. Recall that the third and fourth steps of the TUL and NWY models are estimated by their FKK equivalent.

The results presented in Figs. 3-8 clearly deviate from
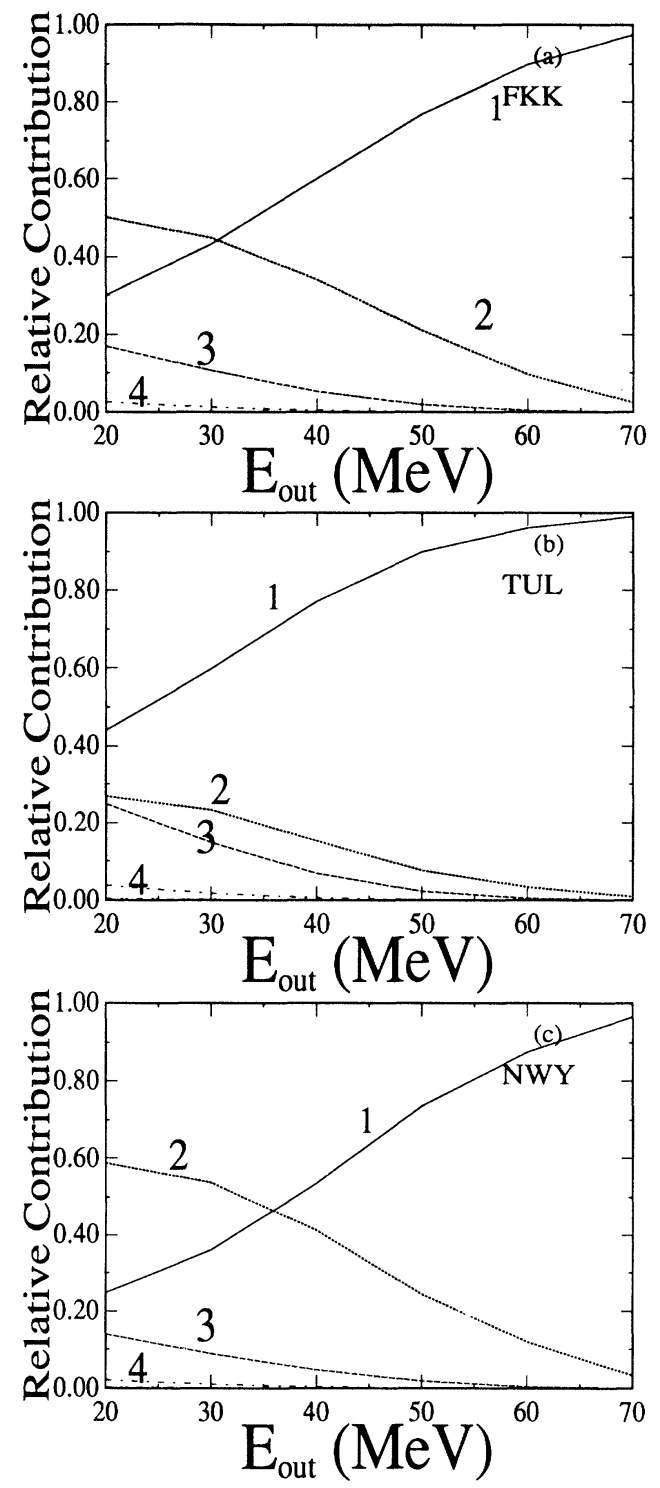

FIG. 7. Angle-integrated relative contribution of each step for $80 \mathrm{MeV}\left(p, p^{\prime}\right)$ on ${ }^{90} \mathrm{Zr}$ for the (a) FKK, (b) TUL, and (c) NWY models.
TABLE I. Partition of the total MSD cross section for 80 $\mathrm{MeV}\left(p, p^{\prime}\right)$ on ${ }^{90} \mathrm{Zr}$.

\begin{tabular}{ccccc}
\hline \hline Model & One step & Two step & Three step & Four step \\
\hline FKK & $66 \%$ & $28 \%$ & $5 \%$ & $1 \%$ \\
NWY & $60 \%$ & $34 \%$ & $5 \%$ & $1 \%$ \\
TUL & $81 \%$ & $12 \%$ & $6 \%$ & $1 \%$ \\
\hline \hline
\end{tabular}

the FKK model analysis as given in Ref. [21]. Although the total angular distributions (i.e., summed over steps) of the latter are in good agreement with the experimental data, the specific contribution to the cross section from the various steps is in disagreement with our conclusions. Fig. 6 (and also 7) of Ref. [21] suggests that the one-step
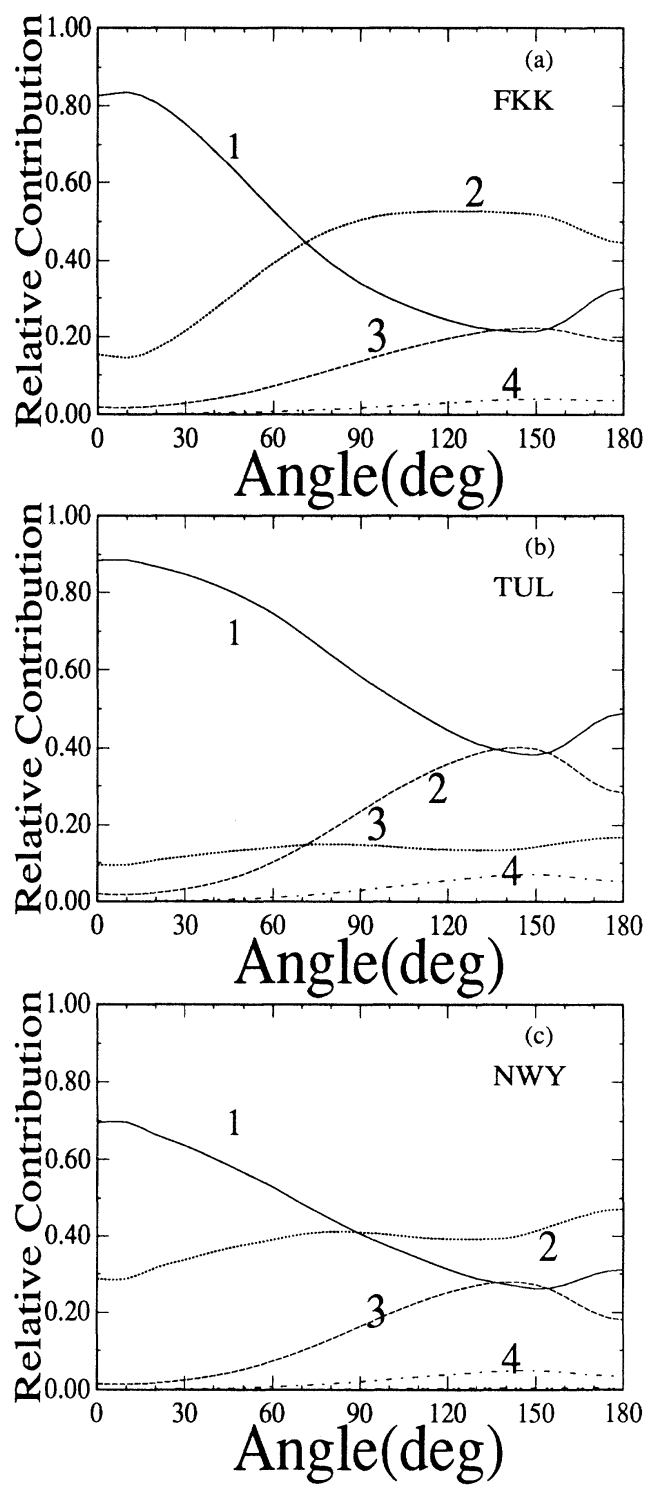

FIG. 8. Energy-integrated relative contribution of each step for $80 \mathrm{MeV}\left(p, p^{\prime}\right)$ on ${ }^{90} \mathrm{Zr}$ for the (a) FKK, (b) TUL, and (c) NWY models. 
cross section is only predominant at the highest outgoing energies at the very forward angles. For example, at the outgoing energy of $60 \mathrm{MeV}$ the one-step cross section falls below the third step already at $80^{\circ}$ and even below the fifth step at $130^{\circ}$. This does not correspond with the behavior of the cross section of Figs. 3-5 of this work. Clearly, the series of cross section contributions converges much more rapidly in our case. Our findings are, however, in line with other, independent, sources such as Refs. [3, 22, 25] and the "one, two, infinity" idea of Ref. [26], where a strong overall predominance of the first and second steps is predicted as well as the correlated fast convergence of the cross section.

The deviations of the results reported in Ref. [21] from ours are partly caused by the use of microscopic DWBA cross sections calculated by DWUCK-4 instead of macroscopic cross sections by ECIS88. However, the main source of the differences are an alternative implementation of the FKK formula and alternative numerical (integration) techniques. To illustrate this, we present in Fig. 9 three different analyses of the angular distribution at the outgoing energy of $60 \mathrm{MeV}$. In Fig. 9(a), we have reproduced Fig. 6(a) of Ref. [21] which basically uses a microscopic approach plus rectangular integration over the intermediate energy. In Fig. 9(b), we present a MSD calculation performed with KAPSIES using a microscopic approach plus numerical integration according to the Simpson rule. Here, we have (in line with [21]) randomly generated particle-hole states with a Nilsson model, grouped them into angular momentum and energy bins, calculated microscopical DWBA cross sections for each particle-hole state with DWUCK-4, and averaged them over the number of states in each bin. Subsequently, these average DWBA cross sections were (instead of our ECIS88 cross sections) offered to the MSD part of KAPSIES where they were processed according to Eqs. (1)-(5). Hence, the difference between Figs. 9(a) and 9(b) is entirely brought about by different numerical implementations of the FKK model and not by alternative DWBA prescriptions; it is seen that our much more accurate integration scheme leads to a completely different mix of MSD steps. The overall fit is roughly the same, although this changes at lower outgoing energies. Obviously, the cross section of Fig. 9(b) converges faster than that of Fig. 9(a) and the differences in magnitude with respect to the third and higher steps are sizable.

The difference between the microscopic approach with DWUCK-4 and the macroscopic approach with ECIS88 is exemplified by Figs. 9(b) and 9(c), where the latter shows the result of a macroscopic approach with Simpson integration. We emphasize that the cross sections in these figures have been calculated by the same MSD program. This time, only the DWBA prescriptions are different. Although the microscopic one-step cross section falls off faster than the one based on ECIS88 DWBA cross sections, the multistep contributions are very similar. This demonstrates that the difference between Figs. 9(a) and 9 (b) due to different numerical algorithms is larger than the difference between the DWBA methods.

To summarize, using a macroscopic approach (as in KAPSIES) is a good approximation to and computation- ally much faster than the microscopic sampling approach of Bonetti et al. It is important to use an accurate numerical scheme for the integration over the intermediate energies. Simpson rule integration (as in KAPSIES) does make a large difference with the rectangular integration by Bonetti et al., the latter method leading to unrealistic high contributions of the higher-order MSD steps. In contrast to previous statements in the literature, the TUL and FKK models converge equally rapidly in terms of the number of MSD steps.
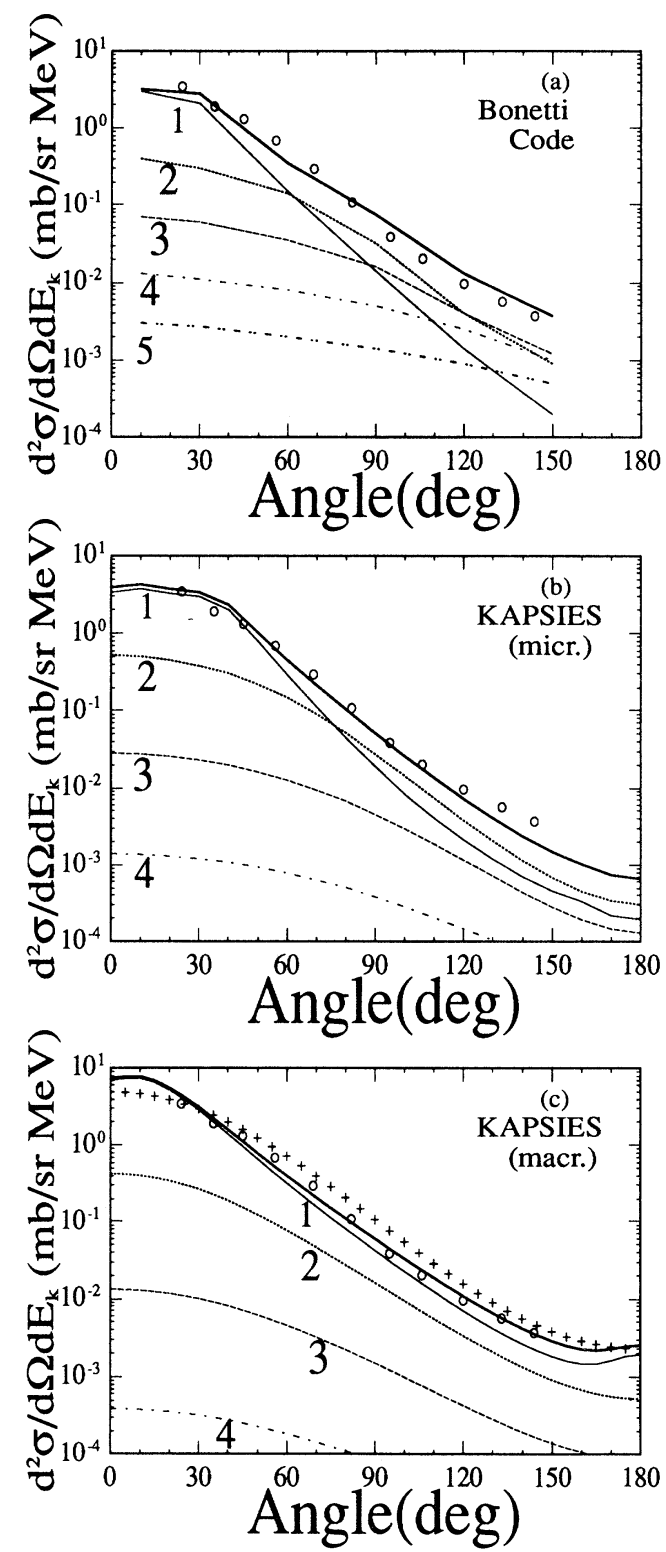

FIG. 9. MSD cross sections according to the FKK model for $80 \mathrm{MeV}\left(p, p^{\prime}\right)$ on ${ }^{90} \mathrm{Zr}$ at an outgoing energy of $60 \mathrm{MeV}$, calculated by (a) the MSD code of Bonetti, using DWUCK-4, (b) KAPSIES, using the method of (a) with shell model states and DWUCK-4, and (c) KAPSIES, standard calculation. 


\section{B. $62 \mathrm{MeV}\left(p, p^{\prime}\right)$ on ${ }^{209} \mathrm{Bi}$}

The typical MSD characteristics of the cross sections are also displayed by the $62 \mathrm{MeV}\left(p, p^{\prime}\right)$ reaction on ${ }^{209} \mathrm{Bi}$. Bertrand and Peelle [27] have published a rather complete set of experimental data for this reaction, including energy-averaged single- and double-differential cross sections at many outgoing energies. Hence, it is possible to test the experimental energy spectrum in addition to the angular distributions. The angular distributions have been calculated quantum mechanically by Tamura et al. [3] and also classically by the generalized exciton model of Iwamoto and Harada [22]. For the angular distributions, we have considered the same outgoing energies as in Ref. [3], namely, 47, 37, and $27 \mathrm{MeV}$, respectively.
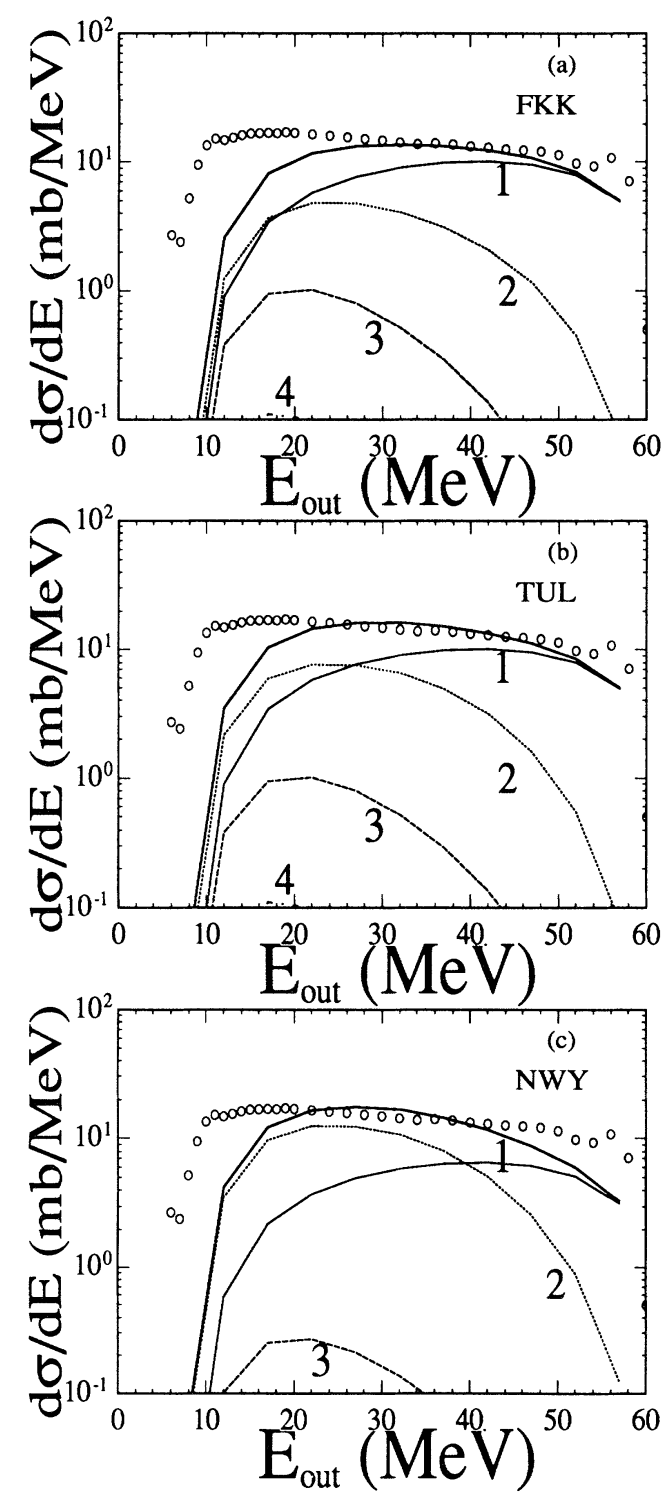

FIG. 10. MSD energy spectrum for $62 \mathrm{MeV}\left(p, p^{\prime}\right)$ on ${ }^{209} \mathrm{Bi}$ for the (a) FKK, (b) TUL, and (c) NWY models. The thick solid line represents the total MSD cross section (summed over steps) and the circles the experimental data.
Again, the distorted waves and the form factors are calculated with the optical potential of Becchetti and Greenlees. The state density parameters we used are $\sigma=4.112$, $P=0.89$, and $a=24.59 \mathrm{MeV}^{-1}\left(g=14.95 \mathrm{MeV}^{-1}\right)$. In Fig. 10 the angle-integrated energy-averaged cross sections are compared with the experimental data. For the deformation parameter, we used $\beta=0.00237$. A striking aspect of these energy spectra is the remarkable similarity between the TUL and FKK two-step cross sections. Furthermore, the MSD process accounts for the total cross section over a wide energy range. The figures also show
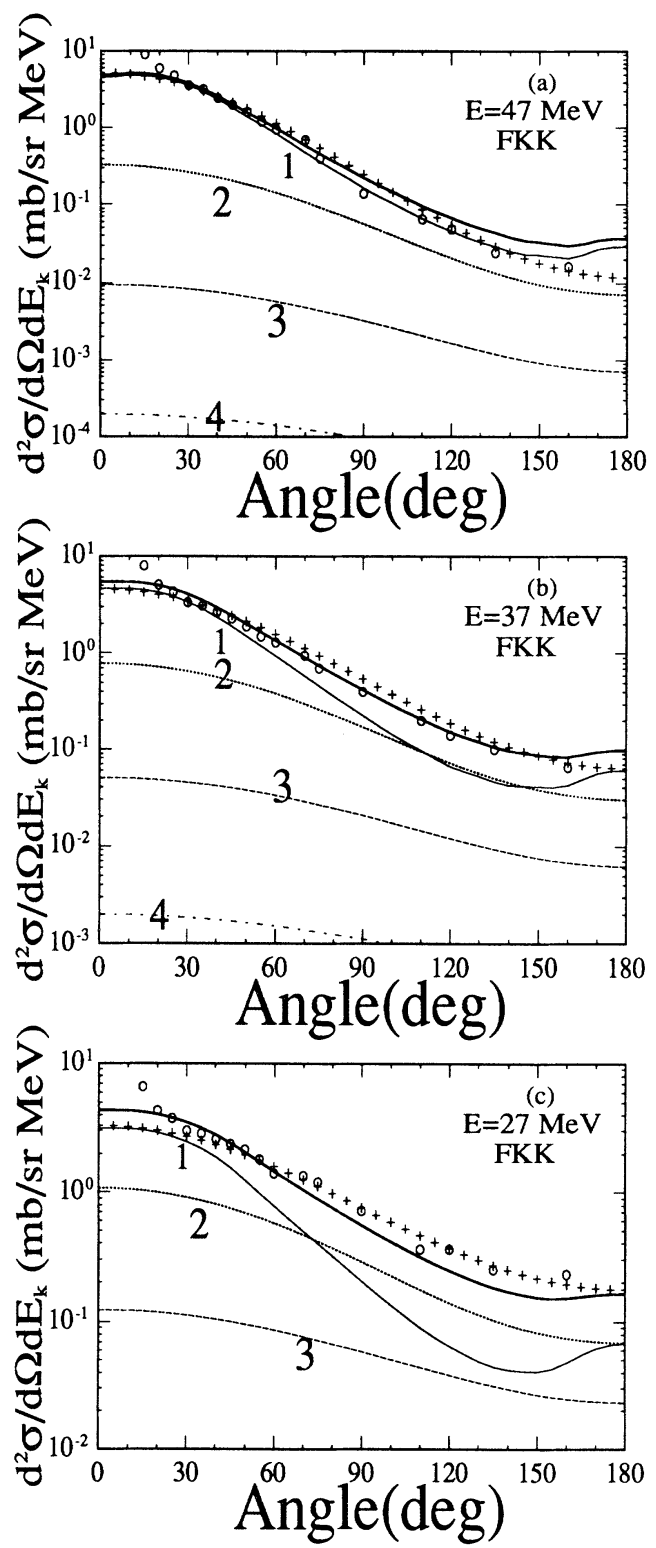

FIG. 11. MSD cross sections according to the FKK model for $62 \mathrm{MeV}\left(p, p^{\prime}\right)$ on ${ }^{209} \mathrm{Bi}$ at outgoing energies of (a) $47 \mathrm{MeV}$, (b) $37 \mathrm{MeV}$, and (c) $27 \mathrm{MeV}$. The thick solid line represents the total MSD cross section (summed over steps), the circles the experimental data, and the pluses the systematics of Kalbach. 
that at the very high outgoing energies (the first few $\mathrm{MeV}$ of excitation energy) collective discrete states are present and the statistical MSD reaction mechanism as presented here is no longer adequate. Below an outgoing energy of $20 \mathrm{MeV}$, the probability for MSD processes to occur is reduced by the competing reaction mechanisms which were already discussed for the $\mathrm{Zr}$ reaction. Related to this is the observation that where the angle-integrated contribution of the third step becomes comparable with the first and second steps, below $15 \mathrm{MeV}$, the total MSD contribution itself has become totally irrelevant. For the
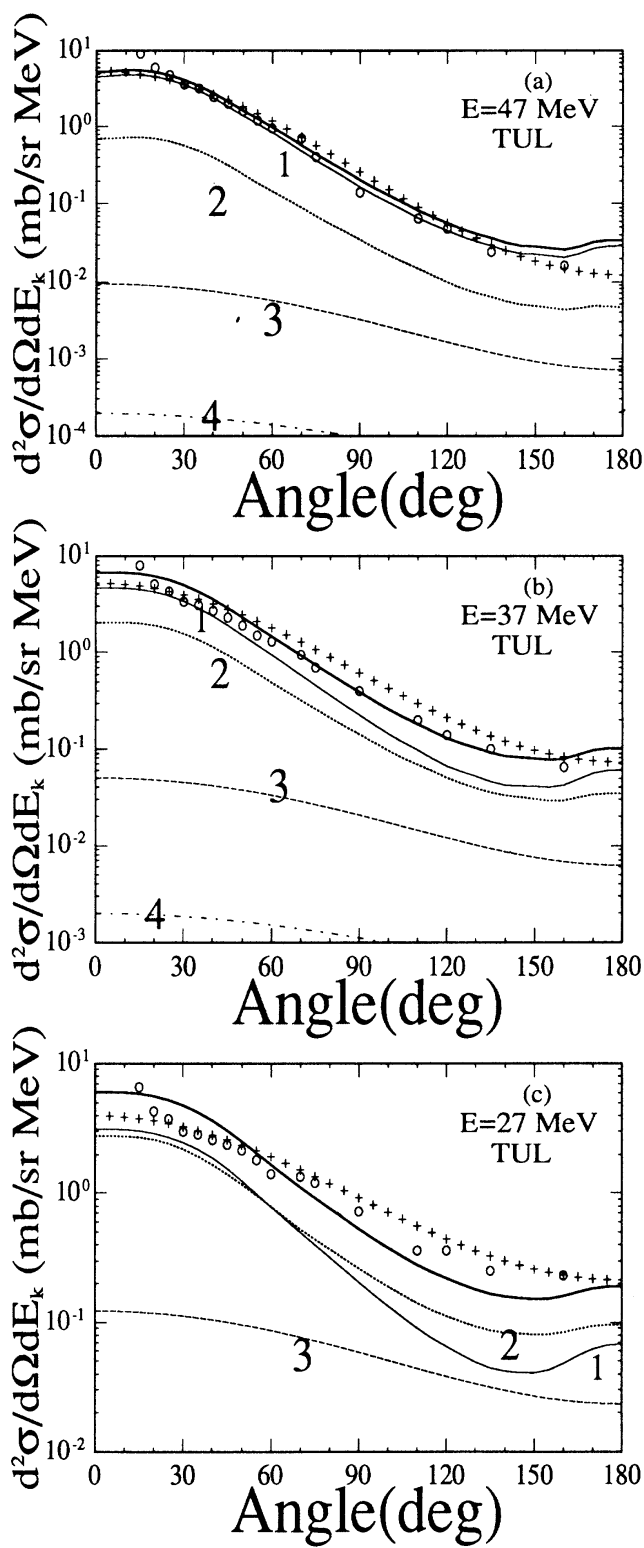

FIG. 12. MSD cross sections according to the TUL model for $62 \mathrm{MeV}\left(p, p^{\prime}\right)$ on ${ }^{209} \mathrm{Bi}$ at outgoing energies of (a) $47 \mathrm{MeV}$, (b) $37 \mathrm{MeV}$, and (c) $27 \mathrm{MeV}$. The thick solid line represents the total MSD cross section (summed over steps), the circles the experimental data, and the pluses the systematics of Kalbach.
NWY model we encounter some difficulties in obtaining a reasonable fit of the high energy tail. We used another value for the deformation parameter, $\beta=0.00190$, but the quality of the FKK and TUL results could not be reproduced for this reaction. Compared with the other two models the two-step contribution is quite large. A possible explanation for this is that the upper limit of the NWY two-step cross section (which we employ throughout this work) is an overestimation of the real value for this reaction.

In Figs. 11-13, the angular distributions for all models
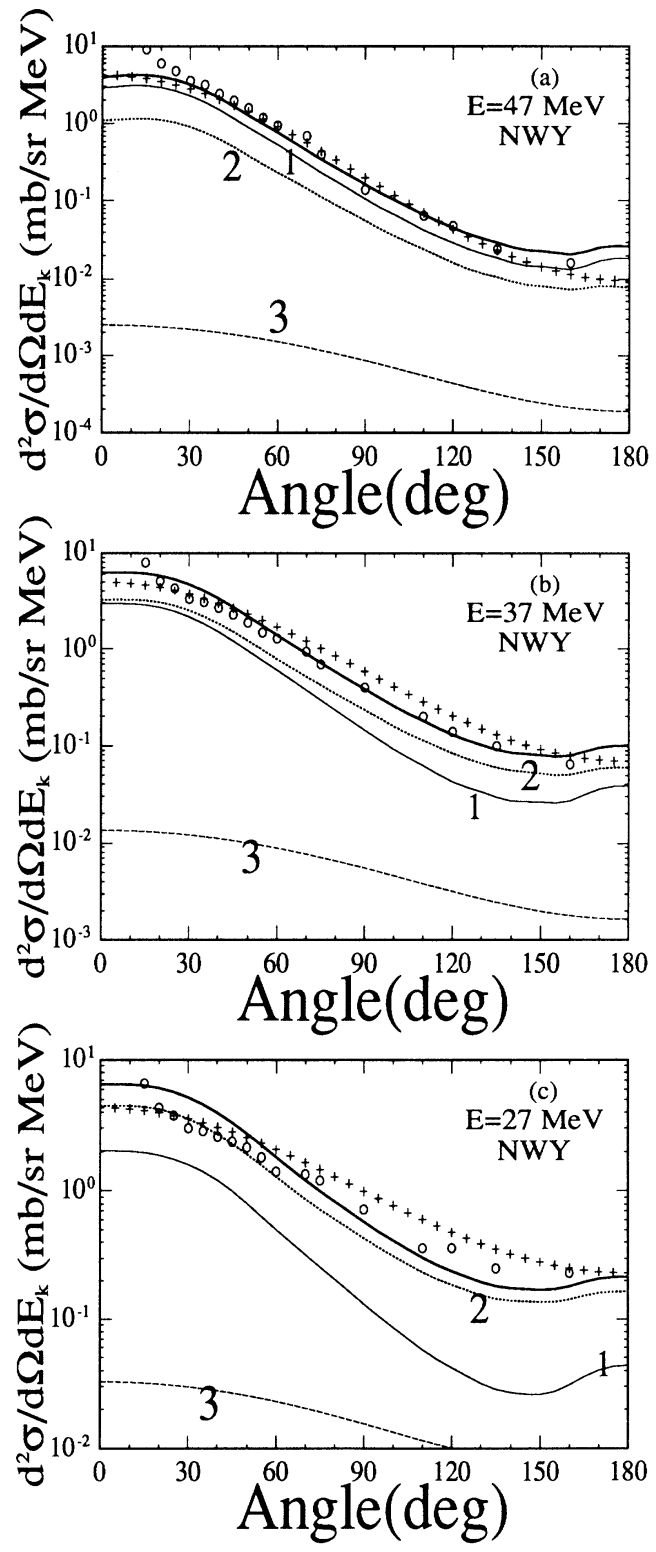

FIG. 13. MSD cross sections according to the NWY model for $62 \mathrm{MeV}\left(p, p^{\prime}\right)$ on ${ }^{209} \mathrm{Bi}$ at outgoing energies of (a) $47 \mathrm{MeV}$, (b) $37 \mathrm{MeV}$, and (c) $27 \mathrm{MeV}$. The thick solid line represents the total MSD cross section (summed over steps), the circles the experimental data, and the pluses the systematics of Kalbach. 
are depicted. The experimental data are averaged over an energy bin of $5 \mathrm{MeV}$. Again, the shape of the angular distributions is quite nicely predicted by all models, with the already mentioned small magnitude problem for the NWY model. Our computational results are comparable in quality with the Kalbach systematics. The two-step cross sections are compared in Fig. 14. For consistency, we took the same value $\beta=0.00237$ for the three models. In this case, the FKK two-step cross section is similar to that of the TUL model (which was already suggested by the energy spectra).

The angle- and energy-integrated relative contributions are displayed in Figs. 15 and 16. Those of the FKK and TUL models are very similar to the corresponding
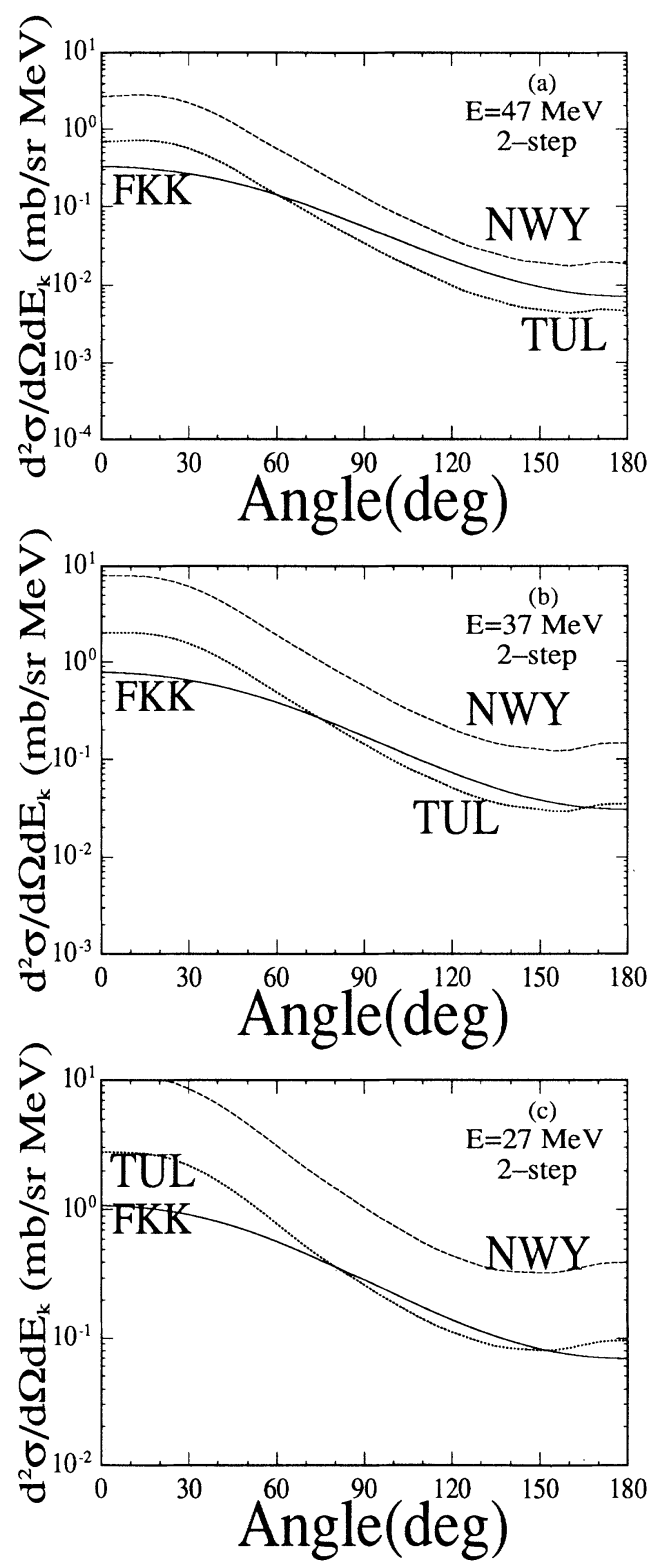

FIG. 14. Two-step cross sections for $62 \mathrm{MeV}\left(p, p^{\prime}\right)$ on ${ }^{209} \mathrm{Bi}$ at outgoing energies of (a) $47 \mathrm{MeV}$, (b) $37 \mathrm{MeV}$, and (c) $27 \mathrm{MeV}$. relative contributions of the ${ }^{90} \mathrm{Zr}$ case. The relative contributions to the total MSD cross section are given in Table II.

In Fig. 1 of Ref. [3], Tamura et al. present an analysis of the same angular distributions. Upon comparison with our Figs. 11-13, we find an almost exactly equivalent partition into one- and two-step contributions and a comparable fit to the double-differential cross section. This suggests (although it certainly needs to be verified for more test cases) that the results are not too sensitive for the particular description of the continuum states. In Ref. [3] (see also Ref. [15]), the reactions to the continuum are described using a spectroscopic density that is expressed in terms of a response function. Accordingly, an estimate for the distribution of the particle-hole states
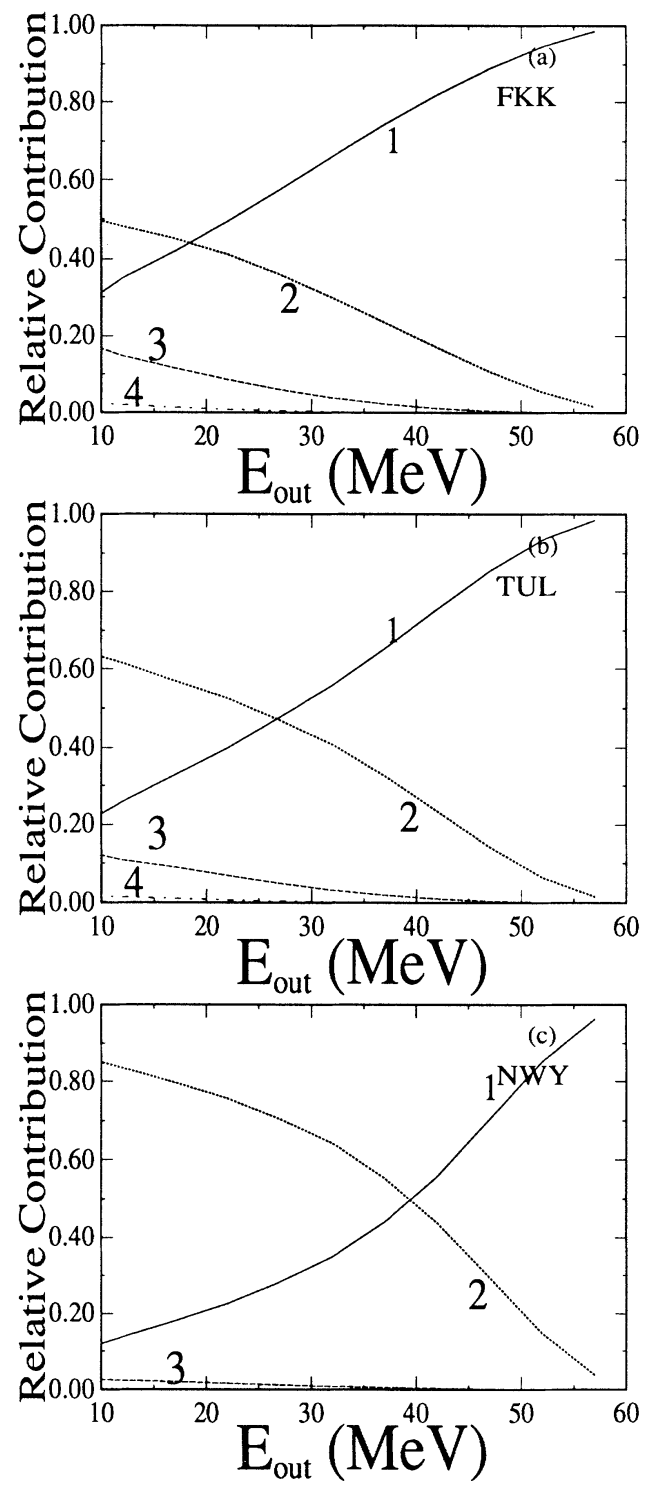

FIG. 15. Angle-integrated relative contribution of each step for $62 \mathrm{MeV}\left(p, p^{\prime}\right)$ on ${ }^{209} \mathrm{Bi}$ for the (a) FKK, (b) TUL, and (c) NWY models. 
over the exact states is obtained. The continuum cross sections are obtained by multiplication of the DWBA cross sections by the spectroscopic density and summation over the total angular momentum. Apparently, our usage of a very simple model for the angular momentum dependent particle-hole state density does not seem to give rise to significant deviations.

The same double-differential cross sections (with slightly different outgoing energies) have been predicted by a generalized exciton model, see Fig. 6 of Ref. [22]. The leading particle approach that is employed by these authors comprises an explicit consideration of the direction and energy of the leading particle at each stage of the multistep process. Therefore, it may be regarded as a semiclassical counterpart of our quantum-mechanical leading-particle statistics approach (FKK model). The
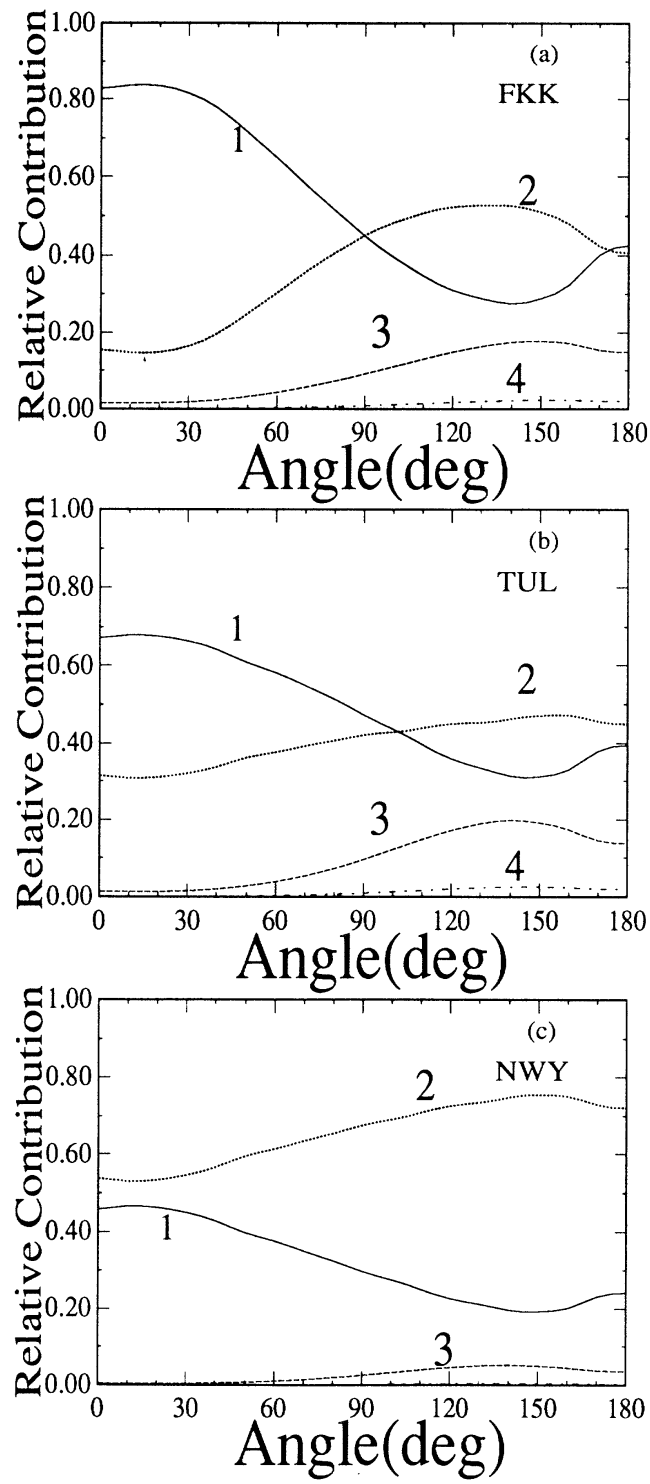

FIG. 16. Energy-integrated relative contribution of each step for $62 \mathrm{MeV}\left(p, p^{\prime}\right)$ on ${ }^{209} \mathrm{Bi}$ for the (a) FKK, (b) TUL, and (c) NWY models.
TABLE II. Partition of the total MSD cross section for $62 \mathrm{MeV}\left(p, p^{\prime}\right)$ on ${ }^{209} \mathrm{Bi}$.

\begin{tabular}{ccccc}
\hline \hline Model & One step & Two step & Three step & Four step \\
\hline FKK & $70 \%$ & $26 \%$ & $4 \%$ & $0 \%$ \\
NWY & $41 \%$ & $58 \%$ & $1 \%$ & $0 \%$ \\
TUL & $61 \%$ & $35 \%$ & $4 \%$ & $0 \%$ \\
\hline \hline
\end{tabular}

results of Ref. [22] confirm a well-known problem of classical preequilibrium models, namely, the incorrect prediction of the angular distribution at backward angles (although generally there is a good agreement with the experimental energy spectrum). This deficiency is seen to be removed by adopting a quantum-mechanical approach.

\section{C. $25.7 \mathrm{MeV}\left(n, n^{\prime}\right)$ on ${ }^{93} \mathrm{Nb}$}

The nucleus ${ }^{93} \mathrm{Nb}$ is known for its pronounced preequilibrium component in the energy spectra of nucleon induced reactions. Therefore, the $25.7 \mathrm{MeV}\left(n, n^{\prime}\right)$ reaction on ${ }^{93} \mathrm{Nb}$ has been subject to an extensive comparison of semiclassical preequilibrium models $[10,23]$. In this compilation, several Hauser-Feshbach models with preequilibrium options, exciton models, and hybrid models have been tested against each other and against experiment with a common set of parameters. Here, we like to compare our quantum-mechanical MSD approaches with the general behavior of the classical models.

The optical model parameters we used are given in Appendix A of Ref. [23]. With ECIS88, we obtained the cross section values $\sigma_{\text {total }}=2918.4 \mathrm{mb}, \sigma_{\text {elas }}=1305.1 \mathrm{mb}$, and $\sigma_{\text {reac }}=1613.3 \mathrm{mb}$, which is in good agreement with the reported values of Ref. [23]. The level density parameters were chosen according to the parametrizations as given in Sec. IV, i.e., $\sigma=3.139, P=1.33$, and $a=11.77$ $\mathrm{MeV}^{-1}\left(g=7.15 \mathrm{MeV}^{-1}\right)$. In Fig. 17, the calculated energy spectra are shown together with the experimental data (which are taken from Ref. [28]). The cross sections were only measured for outgoing energies above 12 $\mathrm{MeV}$. Again, we can conclude that the high energy part of the preequilibrium region is adequately described by the MSD reaction mechanism. In general, the value of the measured cross section increases at lower outgoing energies whereas the MSD contribution remains approximately constant. Here, the MSD process is strongly inhibited by multistep compound, compound, and multiple emission processes. In the figures we have included (dashed curve) the cross section as calculated by the code system GNASH [20] (see Figs. 3D of Ref. [23] or Fig. 3.3 of Ref. [10]) which gives a reliable estimate for the whole outgoing-energy range. The GNASH curve is the result of a combined Hauser-Feshbach + exciton model evaluation.

The values we use for $\beta$ are 0.00693 for the FKK and TUL models and 0.00632 for the NWY model. Obviously, the one-step cross section is almost fully responsible for the explanation of the data. The situation is analogous to that of the ${ }^{209} \mathrm{Bi}$ reaction. Once the discrepancy between the one-step cross section and the total 
cross section becomes sizable (below $10 \mathrm{MeV}$ ), the MSD contribution to the total cross section can be neglected. Hence, our recommendation for a complete quantummechanical analysis of the preequilibrium region of this reaction would consist of continuum one-step direct, multistep compound reactions, and a combination of these two processes.

The calculated angular distributions at outgoing energies of 20,16, and $12 \mathrm{MeV}$ (see Figs. 18-20) display some oscillatory behavior at the backward angles. Closer investigation of the individual DWBA components that construct the energy-average cross section reveals that there is some constructive interference of the angular distributions. Then, these effects are not washed out by the averaging process, in contrast with the other two discussed reactions (cf. Fig. 1). Again, the fit obtained at the highest outgoing energy is the most satisfactory and

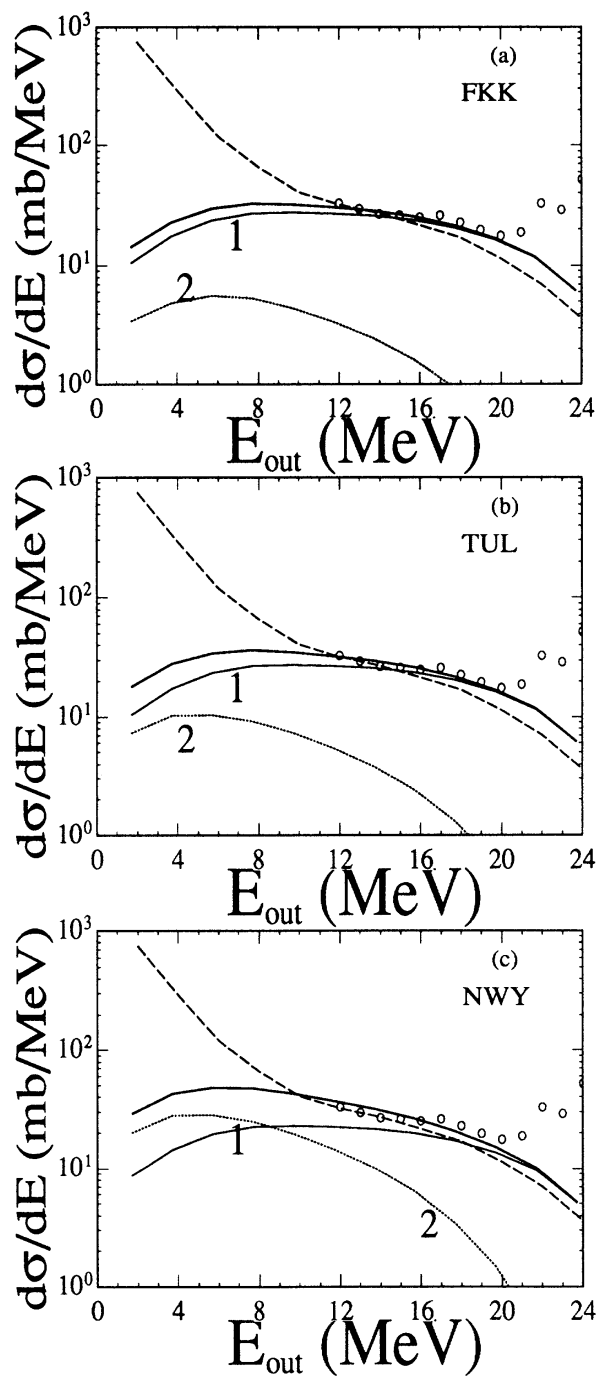

FIG. 17. MSD energy spectrum for $25.7 \mathrm{MeV}\left(n, n^{\prime}\right)$ on ${ }^{93} \mathrm{Nb}$ for the (a) FKK, (b) TUL, and (c) NWY models. The thick solid line represents the total MSD cross section (summed over steps) and the circles the experimental data. The long-dashed line represents the calculation by GNASH. up to $150^{\circ}$ in perfect agreement with the Kalbach systematics. Regrettably, there do not seem to exist data for angles larger than $150^{\circ}$, so that the sudden rise of the cross section beyond this angle cannot be tested. Anyway, it is clear that at these relatively low incident energies the typical quantum-mechanical behavior of the angular distributions becomes more pronounced. In Fig. 3.6 of Ref. [10] (Figs. 15 and 16 of Ref. [23]), some predictions by exciton and hybrid models are presented. In most of these models, refraction and finite-size effects are included as corrections for the semiclassical treatment of the preequilibrium process. Nevertheless, the angu-
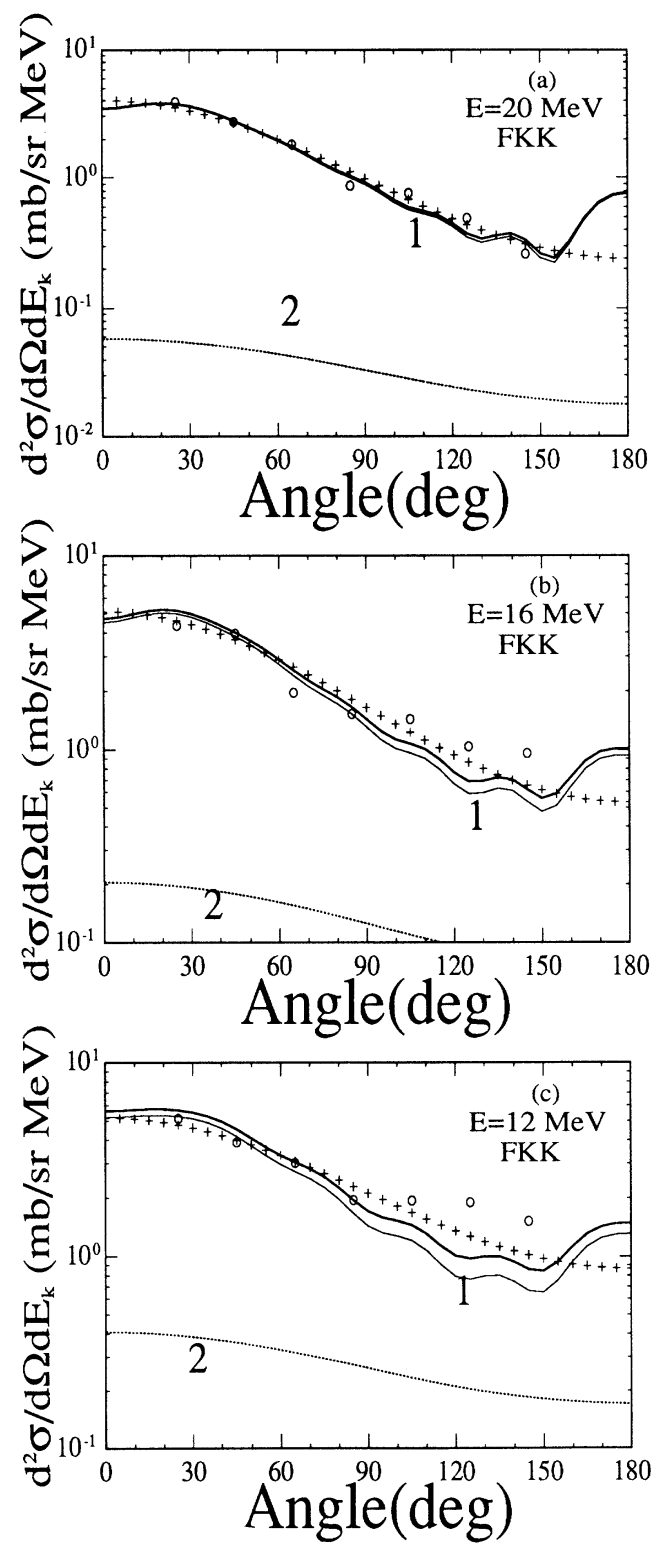

FIG. 18. MSD cross sections according to the FKK model for $25.7 \mathrm{MeV}\left(n, n^{\prime}\right)$ on ${ }^{93} \mathrm{Nb}$ at outgoing energies of (a) 20 $\mathrm{MeV}$, (b) $16 \mathrm{MeV}$, and (c) $12 \mathrm{MeV}$. The thick solid line represents the total MSD cross section (summed over steps), the circles the experimental data, and the pluses the systematics of Kalbach. 
lar distributions as predicted by the semiclassical models still exhibit a systematic underestimation of the experimental data at backward angles, implying that further empirical adjustments are needed. Consequently, at the highest outgoing energies the quantum-mechanical approaches as presented here are better than, and at lower outgoing energies comparable to, the semiclassical analyses of this reaction.

In Fig. 21, we have compared the two-step cross sections for the common value $\beta=0.00693$. Clearly, the convolution structure of the FKK cross section accounts
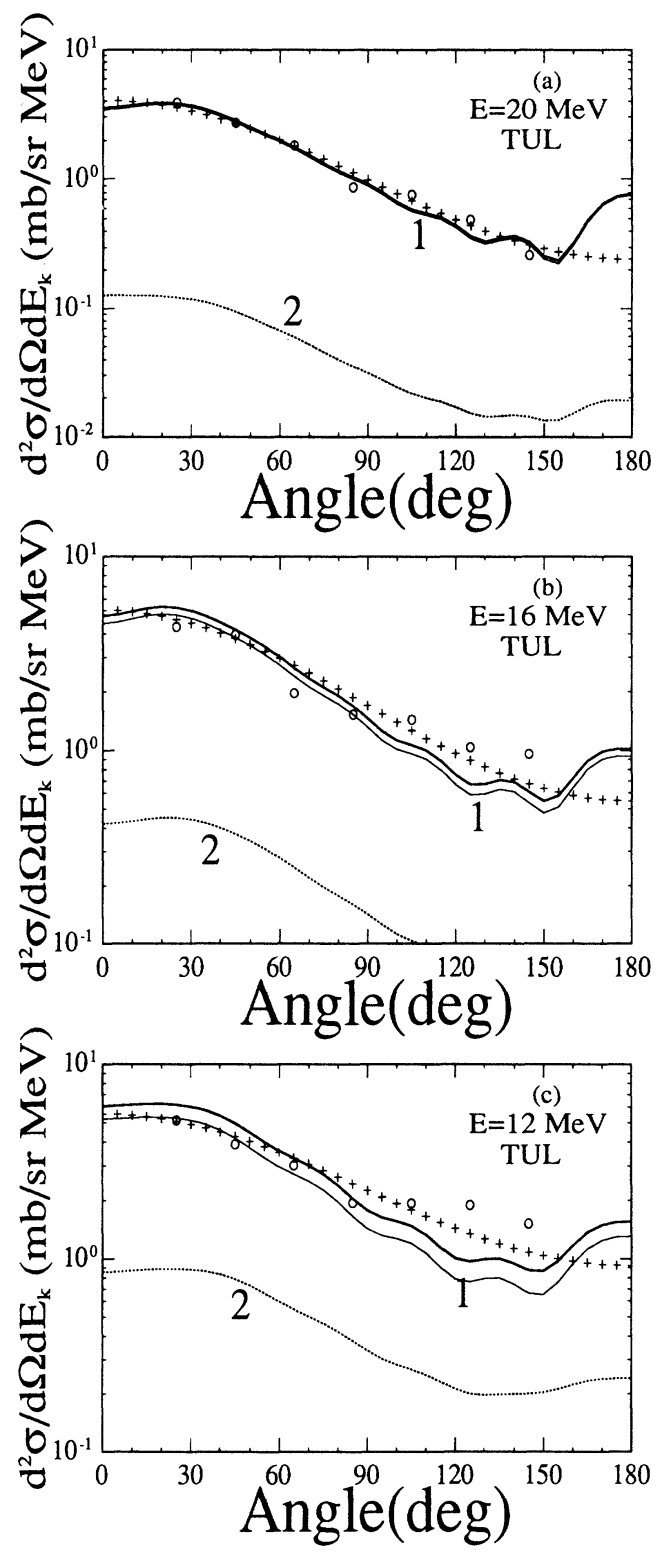

FIG. 19. MSD cross sections according to the TUL model for $25.7 \mathrm{MeV}\left(n, n^{\prime}\right)$ on ${ }^{93} \mathrm{Nb}$ at outgoing energies of (a) 20 $\mathrm{MeV}$, (b) $16 \mathrm{MeV}$, and (c) $12 \mathrm{MeV}$. The thick solid line represents the total MSD cross section (summed over steps), the circles the experimental data, and the pluses the systematics of Kalbach. for the absence of the oscillatory behavior of the two-step angular distribution. For the TUL and NWY models, these effects are still present (although less prominent than in the one-step cross section). In Table III, the partition of the MSD cross section into the various steps is given.

\section{CONCLUSIONS}

The various existing quantum models for multistep direct reactions (FKK, TUL, NWY) have been implemented in one computer code system called KAPSIES.
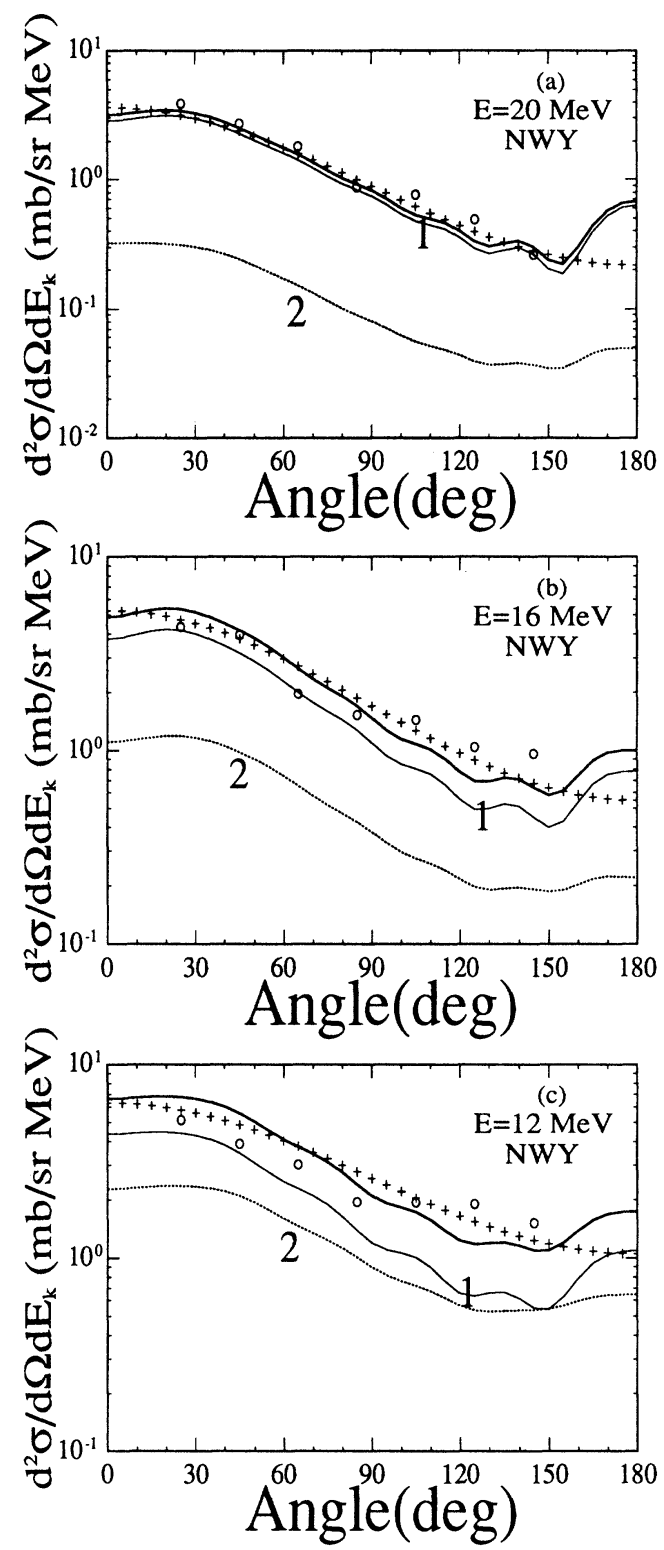

FIG. 20. MSD cross sections according to the NWY model for $25.7 \mathrm{MeV}\left(n, n^{\prime}\right)$ on ${ }^{93} \mathrm{Nb}$ at outgoing energies of (a) 20 $\mathrm{MeV}$, (b) $16 \mathrm{MeV}$, and (c) $12 \mathrm{MeV}$. The thick solid line represents the total MSD cross section (summed over steps), the circles the experimental data, and the pluses the systematics of Kalbach. 
This work is the first to perform a consistent practical comparison of these models, on the basis of the same set of parameters and the same set of numerical techniques for the MSD calculations. The method of implementation of the MSD models has been discussed in Sec. IV. It appears that the FKK and TUL models are rather simple to implement on a computer, whereas the implementation of the NWY model is complicated and certain approximations are unavoidable due to the lack of microscopic information.

All our calculations are based on the adjustment of only one parameter, the deformation parameter $\beta$, which is taken in the computations to be independent of $l$. We have fitted the $\beta$ at the angle-integrated cross section
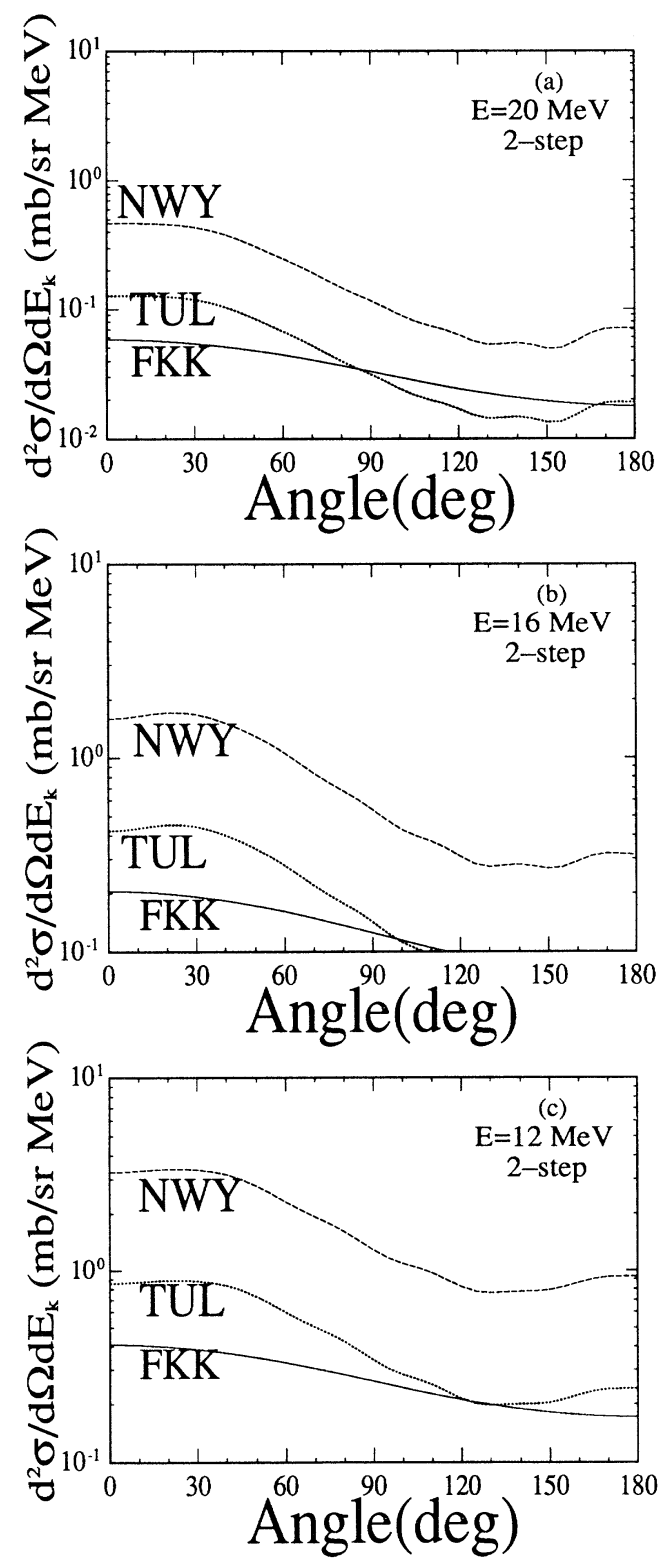

FIG. 21. Two-step cross sections for $25.7 \mathrm{MeV}\left(n, n^{\prime}\right)$ on ${ }^{93} \mathrm{Nb}$ at outgoing energies of (a) $20 \mathrm{MeV}$, (b) $16 \mathrm{MeV}$, and (c) $12 \mathrm{MeV}$.
TABLE III. Partition of the total MSD cross section for $25.7 \mathrm{MeV}\left(n, n^{\prime}\right)$ on ${ }^{93} \mathrm{Nb}$.

\begin{tabular}{lcccc}
\hline \hline Model & One step & Two step & Three step & Four step \\
\hline FKK & $88 \%$ & $12 \%$ & $0 \%$ & $0 \%$ \\
NWY & $57 \%$ & $43 \%$ & $0 \%$ & $0 \%$ \\
TUL & $81 \%$ & $19 \%$ & $0 \%$ & $0 \%$ \\
\hline \hline
\end{tabular}

at the highest outgoing energy where it can reasonably be assumed that the whole cross section stems from the MSD process. Various experimental cross sections in the preequilibrium region of the nuclear reaction spectrum have been analyzed with all MSD models, viz., $80 \mathrm{MeV}$ $\left(p, p^{\prime}\right)$ on ${ }^{90} \mathrm{Zr}, 62 \mathrm{MeV}\left(p, p^{\prime}\right)$ on ${ }^{209} \mathrm{Bi}$, and $25.7 \mathrm{MeV}$ $\left(n, n^{\prime}\right){ }^{93} \mathrm{Nb}$.

The comparison that we have carried out leads to the following conclusions.

A good description of the whole high energy tail of the preequilibrium energy spectrum is achieved by all MSD models. At low outgoing energies, the measured cross section is underestimated by the MSD calculation due to expected competing reaction mechanisms such as multiple emission and MSD-MSC crossover reactions (in which the multistep compound chain is fed after one or two direct steps).

Another (and arguably the most important) test case for the MSD models is the angular distribution. The calculated MSD angular distributions are, again for all MSD models, in very good agreement with the experimental data. It has been shown that quantum-mechanical models perform as good as the systematics of Kalbach, and in some cases even better, and provide a clear improvement of the angular distributions as estimated by the classical preequilibrium models. The latter generally underpredict the experimental data at backward angles.

Our analysis also shows that the computational results of the various MSD models, although physically very different, are roughly of the same quality with respect to experiment. Leading-particle statistics (randomness of DWBA matrix elements, as used in the FKK model) is clearly a stronger assumption-and therefore leads to simpler models - than residual-system statistics (random configuration mixing), but appears to be adequate for the analysis of this type of data. Therefore, on the basis of cross section data it is not possible to discriminate between the various MSD models. It would be interesting, however, to extend the current analysis to polarization data.

Our calculations show that at the energies considered only the first two steps of the MSD process contribute significantly to the preequilibrium cross section. When the third step becomes important, the MSD cross section is already inhibited by competing reaction mechanisms.

Thus, the quantum-mechanical MSD models yield a better description of the experimental data than the semiclassical preequilibrium models. Furthermore, the simplest quantum MSD model (FKK) performs as good as the more complicated ones (NWY and TUL). Hence, for practical reasons, we would recommend the FKK model for nuclear data applications. 
[1] A. J. Koning and J. M. Akkermans, Ann. Phys. 208, 216 (1991).

[2] H. Feshbach, A. Kerman, and S. Koonin, Ann. Phys. 125, 429 (1980).

[3] T. Tamura, T. Udagawa, and H. Lenske, Phys. Rev. C 26, 379 (1982).

[4] H. Nishioka, H. A. Weidenmüller, and S. Yoshida, Ann. Phys. 183, 166 (1988).

[5] H. Nishioka and H. A. Weidenmüller, Phys. Lett. B 203, 1 (1988).

[6] L. Avaldi, R. Bonetti, and L. C. Milazzo, Phys. Lett. 94B, 463 (1980).

[7] R. Bonetti, M. Camnasio, L. C. Milazzo, and P. E. Hodgson, Phys. Rev. C 24, 71 (1981).

[8] A. Marcinkowski, R. W. Finlay, J. Rapaport, P. E. Hodgson, and M. B. Chadwick, Nucl. Phys. A501, 1 (1989).

[9] J. M. Akkermans, H. Gruppelaar, and G. Reffo, Phys. Rev. C 22, 73 (1980).

[10] H. Gruppelaar, P. Nagel, and P. E. Hodgson, Riv. Nuovo Cimento 9, 1 (1986).

[11] C. Kalbach, Phys. Rev. C 37, 2350 (1988).

[12] F. C. Williams, Nucl. Phys. A166, 231 (1971).

[13] S. M. Austin, in Proceedings of the Conference on the $(p, n)$ Reaction and the Nucleon-Nucleon Force, edited by C. D. Goodman et al. (Plenum, New York, 1980), p. 203.

[14] R. Bonetti and C. Chiesa, private communication.

[15] T. Tamura, T. Udagawa, and M. Benhamou, Comput. Phys. Commun. 29, 391 (1983).

[16] J. Raynal, Computing as a Language of Physics, Trieste, 1971 (IAEA, Vienna, 1972); private communication. The code ECIS-88 was obtained from the NEA Data Bank, Paris.
[17] G. R. Satchler, Direct Nuclear Reactions (Oxford University Press, New York, 1983).

[18] W. Dilg, W. Schantl, H. Vonach, and M. Uhl, Nucl. Phys. A217, 269 (1973).

[19] H. Gruppelaar, in Proceedings of the IAEA Advisory Group Meeting on Basic and Applied Problems on $\mathrm{Nu}$ clear Level Densities, edited by M. R. Bhat (Brookhaven National Laboratory, Brookhaven, 1983), p. 143.

[20] E. D. Arthur, in Proceedings of the Workshop on Applied Nuclear Theory and Nuclear Model Calculations for $\mathrm{Nu}$ clear Technology Applications, edited by M. K. Mehta and J. I. Schmidt (IAEA, Trieste, 1988), p. 676.

[21] A. A. Cowley, A. van Kent, J. J. Lawrie, S. V. Förtsch, D. M. Whittal, J. V. Pilcher, F. D. Smit, W. A. Richter, R. Lindsay, I. J. van Heerden, R. Bonetti, and P. E. Hodgson, Phys. Rev. C 43, 678 (1991).

[22] A. Iwamoto and K. Harada, Nucl. Phys. A419, 472 (1984).

[23] H. Gruppelaar and P. Nagel, Newsletter of the NEA Data Bank, NEANDC-204 "U" (1985).

[24] H. Nishioka, H. A. Weidenmüller, and S. Yoshida, Z. Phys. A 336, 197 (1990).

[25] C. Costa, J. M. Akkermans, and H. Gruppelaar, Phys. Rev. C 28, 587 (1983).

[26] H. C. Chiang and J. Hüfner, Nucl. Phys. A349, 466 (1980).

[27] F. E. Bertrand and R. W. Peelle, Oak Ridge National Laboratory Report ORNL-4638, 1971.

[28] A. Marcinkowski, R. W. Finlay, G. Sanders-Pehrson, C. E. Brient, R. Kurup, S. Mellema, A. Meigoni, and R. Tailor, Nucl. Sci. Eng. 83, 13 (1983). 